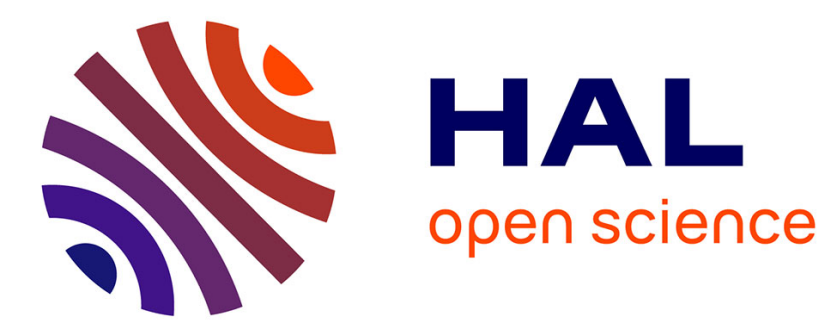

\title{
Moving Kriging reconstruction for high-order finite volume computation of compressible flows
}

\author{
Jean-Camille Chassaing, Xesús Nogueira, Sofiane Khelladi
}

\section{To cite this version:}

Jean-Camille Chassaing, Xesús Nogueira, Sofiane Khelladi. Moving Kriging reconstruction for highorder finite volume computation of compressible flows. Computer Methods in Applied Mechanics and Engineering, 2013, 253, pp.463-478. 10.1016/j.cma.2012.08.016 . hal-01459738

\section{HAL Id: hal-01459738 \\ https://hal.science/hal-01459738}

Submitted on 3 Sep 2020

HAL is a multi-disciplinary open access archive for the deposit and dissemination of scientific research documents, whether they are published or not. The documents may come from teaching and research institutions in France or abroad, or from public or private research centers.
L'archive ouverte pluridisciplinaire HAL, est destinée au dépôt et à la diffusion de documents scientifiques de niveau recherche, publiés ou non, émanant des établissements d'enseignement et de recherche français ou étrangers, des laboratoires publics ou privés. 


\title{
Moving Kriging reconstruction for high-order finite volume computation of compressible flows
}

\author{
Jean-Camille Chassaing ${ }^{\mathrm{a}, *}$, Xesús Nogueira ${ }^{\mathrm{b}}$, Sofiane Khelladi ${ }^{\mathrm{c}}$ \\ a Institut Jean Le Rond d'Alembert, UPMC Univ Paris 06, UMR 7190, F-75005 Paris, France \\ ${ }^{\mathrm{b}}$ Grupo de Métodos Numéricos en Ingeniería, GMNI, Universidade da Coruña, A Coruña, Spain \\ ${ }^{\mathrm{c}}$ Laboratoire de Dynamique des Fluides, Arts et Métiers ParisTech, 151 Boulevard de l'Hôpital, 75013 Paris, France
}

\begin{abstract}
This paper describes the development of a high-order finite volume method for the solution of compress-ible viscous flows on unstructured meshes. The novelty of this approach is based on the use of moving Kriging shape functions for the computation of the derivatives in the numerical flux reconstruction step at the cell faces. For each cell, the successive derivatives of the flow variables are deduced from the inter-polation function constructed from a compact stencil support for both Gaussian and quartic spline cor-relation models. A particular attention is paid for the study of the influence of the correlation parameter onto the accuracy of the numerical scheme. The effect of the size of the moving Kriging stencil is also investigated. Robustness and convergence properties are studied for various inviscid and viscous flows. Results reveal that the moving Kriging shape function can be considered as an interesting alterna-tive for the development of high-order methodology for complex geometries.
\end{abstract}

\section{Introduction}

Nowadays computational fluid dynamics (CFD) is routinely used for many applications in aerodynamics, hydromechanics and aerospace. Achieving high-order of accuracy on unstructured grids, and thus obtaining a better solution than second-order schemes for the same grid resolution, should improve the prediction of complex flow features. Such issue can be addressed using several numerical approaches [1], among others, the high-order continuous finite element method (FEM), the discontinuous Galerkin (DG) method and the spectral volume method (SVM). For instance, significant reduction of spurious entropy generation can be obtained using a third-order discretization for the solution of an inviscid steady flow past a subsonic airfoil $[2,3]$.

The most popular approaches employed to construct high-order finite-volume schemes for hyperbolic conservation laws on unstructured grids are the $k$-exact least-squares reconstruction [4-11] and the ENO [12,5,13] or WENO [14-17] reconstructions. However, it is well known that the accuracy of a high-order finite volume method depends strongly in the computation of the gradients and higher-order derivatives. As a consequence, the selection of the approximation or interpolation methodology to the computation of the derivatives is very important, and it greatly influences

\footnotetext{
* Corresponding author.

E-mail addresses: jean-camille.chassaing@upmc.fr (J.-C. Chassaing),xnogueira@ udc.es (X. Nogueira), sofiane.khelladi@paris.ensam.fr (S. Khelladi).
}

the properties of the numerical scheme. The use of meshless approximations methods seems to be an promising alternative to $k$-exact and WENO reconstructions since these approaches are designed to work with scattered data $[18,19]$. Thus, they can be straightforwardly used on unstructured grids for problems in complex geometries. Cueto-Felgueroso et al. [20] and Nogueira et al. [21] adopted a moving least squares (MLS) reproducing kernel method for the computation of the derivatives required for the reconstruction of the variables inside the control volumes. This approach, namely the finite-volume MLS (FV-MLS) method, has been successfully employed for solving wave propagation problems as the linearized Euler equations [22], and also not purely wave propagation problems, such as Navier-Stokes equations [23,24]. If we only consider the convective terms, MLS reconstruction may be regarded as a higher-order extension of classical second-order finite volume methods since they serve to compute the extra terms involved in the Taylor expansion used in the reconstruction step [11], similarly to the $k$-exact least-squares reconstruction [4]. An additional advantage of this approach is the treatment of diffusive terms, that are computed directly at integration points. This allows a very accurate, centered, discretization of these terms. The resulting savings in coding efforts are therefore significant compared to the WENO reconstruction. Suitable interpolation kernels must be employed for the computation of the MLS shape functions in order to maintain the robustness and the accuracy of the method. Most and Bucher $[25,26]$ developed regularized MLS weighting functions in the context of the element-free Galerkin method in structural analysis. 
However, MLS-based reproducing kernel method is not the only possible choice. There are many other candidates to be selected as the interpolation/approximation technique [27-29]. Cite, for instance, the radial polynomial interpolation method (RPIM) [30,31], the local maximum entropy (LME) approach [32] and the moving Kriging (MK) interpolation [27,29]. The latter can be regarded as a regression technique to interpolate spatially and temporally correlated data [33]. Kriging models, whose regression coefficients are based on the best linear unbiased estimation, are also widely used to construct accurate global approximations in multidisciplinary design optimization $[34,35]$. In order to overcome the difficulty of accurately imposing essential boundary conditions in element-free Galerkin (EFG) methods, Gu [36] proposed to build EFG shape functions using moving Kriging (MK) interpolation instead of MLS approximations for solving the weak form of the steady-state heat conduction problem. This approach was then deployed for various applications in solid mechanics [29,37-43] including both static and dynamic structural analysis. Moving Kriging interpolation requires the choice of a correlation parameter which depends from the problem of interest. This is very similar to the selection of the shape parameters for MLS reconstructions [21]. Recently, Bui and Nguyen [43] used a MK-based meshfree method for the eigenvalue analysis of Kirchhoff thin plate structures with complex geometric shapes. Improved mesh-free approximations were developed by Shaw et al. [44] using a Kriging based errorreproducing and interpolating kernel method (ERIKM). In order to prevent numerical instabilities, the derivatives of the basis functions are not computed directly but using a polynomial reproducing condition.

In this work, we aim to show the feasibility of using MK shape functions for building a novel high-order finite volume method for the solution of compressible flows on unstructured grids. To this end, the successive derivatives of the MK basis are computed directly based on the choice of the expected formal order of accuracy. Then, numerical fluxes are evaluated in the similar way as for other high-order reconstruction schemes using Taylor expansions. A particular attention will be paid for the study of the influence of the correlation parameter on the solution accuracy and robustness.

The outline of this paper is organized as follows. Section 2 briefly describes the finite volume formulation of the governing equations. The high-order discretization algorithm based on moving Kriging reconstruction is detailed in Section 3. Section 4 presents a deep investigation of the proposed scheme for both smooth and transonic inviscid flows. The application of the FVMK approach to viscous unsteady flows is presented in Section 5 and concluding remarks are drawn in Section 6.

\section{Governing equations and finite volume formulation}

The conservative form of the two-dimensional Navier-Stokes equations can be written in Cartesian coordinates as

$\frac{\partial \mathbf{Q}}{\partial t}+\frac{\partial\left(\mathbf{F}_{x}-\mathbf{G}_{x}\right)}{\partial x}+\frac{\partial\left(\mathbf{F}_{y}-\mathbf{G}_{y}\right)}{\partial y}=\mathbf{0}$,

where the vector of conservative variables $\mathbf{Q}$, the vector of the inviscid fluxes $\mathbf{F}=\left(\mathbf{F}_{x}, \mathbf{F}_{y}\right)^{\mathrm{T}}$ and the vector of the viscous fluxes $\mathbf{G}=\left(\mathbf{G}_{x}, \mathbf{G}_{y}\right)^{\mathrm{T}}$ are given by

$$
\mathbf{Q}=\left(\begin{array}{c}
\rho \\
\rho u \\
\rho v \\
E
\end{array}\right), \quad \mathbf{F}_{x}(\mathbf{Q})=\left(\begin{array}{c}
\rho u \\
\rho u^{2}+p \\
\rho u v \\
u(E+p)
\end{array}\right), \mathbf{F}_{y}(\mathbf{Q})=\left(\begin{array}{c}
\rho v \\
\rho u v \\
\rho v^{2}+p \\
v(E+p)
\end{array}\right) \text {, }
$$

$$
\begin{gathered}
\mathbf{G}_{x}(\mathbf{Q})=\mu\left(\begin{array}{c}
0 \\
2 u_{x}-\frac{2}{3}\left(u_{x}+v_{y}\right) \\
v_{x}+u_{y} \\
u\left(2 u_{x}-\frac{2}{3}\left(u_{x}+v_{y}\right)\right)+v\left(v_{x}+u_{y}\right)+\frac{c_{p}}{P_{r}} T_{x}
\end{array}\right), \\
0 \\
\mathbf{G}_{y}(\mathbf{Q})=\mu\left(\begin{array}{c}
v_{x}+u_{y} \\
2 v_{y}-\frac{2}{3}\left(u_{x}+v_{y}\right) \\
u\left(v_{x}+u_{y}\right)+v\left(2 v_{y}-\frac{2}{3}\left(u_{x}+v_{y}\right)\right)+\frac{c_{p}}{P_{r}} T_{y}
\end{array}\right) .
\end{gathered}
$$

Here, $\rho$ is the density, $u, v$ are the $x$-wise and $y$-wise components of the velocity vector, and $p, E$ denote the pressure and the total energy respectively, $\mu$ denotes the dynamic molecular viscosity, $T$ is the temperature, $P_{r}$ is the Prandtl number and $C_{p}$ denotes the specific heat at constant temperature. The Sutherland's law is employed to compute the dynamic viscosity

$\mu=\mu_{\infty} \frac{T+S_{0}}{T_{\infty}+S_{0}}\left(\frac{T}{T_{\infty}}\right)^{1.5}$

where $\mu_{\infty}$ and $T_{\infty}$ are the free-stream viscosity and temperature and $S_{0}=110.4 \mathrm{~K}$. The equation of state for an ideal gas is used to close the system of equations

$p=(\gamma-1)\left(E-\frac{\rho}{2}\left(u^{2}+v^{2}\right)\right)$

where the ratio of specific heats is $\gamma=1.4$. The governing equations are discretized using a cell-centered finite-volume formulation on an arbitrary unstructured grid. The problem domain $\Omega$ is divided into non-overlapping triangular cells $\Omega_{i}$ whose number of face is denoted by $n_{f}$. Since the present study is restricted to the particular case of non-moving and non-deforming meshes, the semi-discrete finite-volume formulation of Eq. (1) reads

$\left|\Omega_{i}\right| \frac{\partial \overline{\mathbf{Q}}_{i}}{\partial t}+\sum_{\ell=1}^{n_{f}} \int_{\Gamma_{\ell}}[\mathbf{F}(\mathbf{Q}(\boldsymbol{x}, t))-\mathbf{G}(\mathbf{Q}(\boldsymbol{x}, t))] \cdot \hat{\mathbf{n}} \mathrm{d} s=0$,

where $\overline{\mathbf{Q}}_{i}$ denotes the cell-averaged value of the solution

$\overline{\mathbf{Q}}_{i}=\frac{1}{\left|\Omega_{i}\right|} \int_{\Omega_{i}} \mathbf{Q}(\boldsymbol{x}, t) \mathrm{d} s$.

The flux integrals involved in (6) must be computed in such a way that the order of the flux integration should be at least equal than the accuracy of the reconstruction of the solution [11]. In this work, we employ Gauss-Legendre quadratures. Therefore, a quadrature rule with $n_{q}$ points integrates exactly a polynomial of degree $2 n_{q}-1$. For instance, the integral involving the inviscid fluxes (6) is approximated as

$\int_{\Gamma_{\ell}} \mathbf{F}(\mathbf{Q}(\boldsymbol{x}, t)) \cdot \hat{\mathbf{n}} \mathrm{d} s \approx\left|\Gamma_{\ell}\right| \sum_{q=1}^{n_{q}} w_{q} \mathbf{F}\left(\mathbf{Q}\left(\boldsymbol{x}_{q}, t\right)\right) \cdot \hat{\mathbf{n}}$,

where $\boldsymbol{x}_{q}$ denotes the quadrature point with quadrature weight $w_{q}$. A single quadrature point situated at mid-side of the face with unit weight is considered for linear reconstruction. Two equallyweighted quadrature points per face are employed for the case of quadratic and cubic reconstructions.

The spatially discretized form of the semi-discrete finite volume Eq. (6) can be written in short form as

$\frac{d \overline{\mathbf{Q}}_{i}}{d t}=-\frac{1}{\left|\Omega_{i}\right|} \sum_{\ell=1}^{n_{f}} \sum_{q=1}^{n_{q}}\left|\Gamma_{\ell}\right| w_{q}\left[\mathbf{F}\left(\mathbf{Q}\left(\boldsymbol{x}_{q}, t\right)\right)-\mathbf{G}\left(\mathbf{Q}\left(\boldsymbol{x}_{q}, t\right)\right)\right] \cdot \hat{\mathbf{n}} \equiv \mathbf{R}_{i}(\overline{\mathbf{Q}})$,

where $\mathbf{R}_{i}(\overline{\mathbf{Q}})$ represents the residual for the $i$ th cell. Hereafter the bar symbol will be dropped for sake of clarity. The normal fluxes across each element face in (9) can be evaluated using any suitable numerical flux $\mathbf{F}^{\text {num }}$ based on the right and left Riemann states of cell face 
$\mathbf{F}(\mathbf{Q}) \cdot \hat{\mathbf{n}} \approx \mathbf{F}^{\text {num }}\left(\mathbf{Q}_{L}, \mathbf{Q}_{R}, \hat{\mathbf{n}}\right)$,

where subscripts $L$ and $R$ indicate the states of the flow properties at the right- and left-side of the cell face. In this work, we employ the HLLC approximate Riemann solver $[45,46]$ which is based on the approximation of the Riemann fan with two intermediate states $\mathbf{Q}_{L}^{*}$ and $\mathbf{Q}_{R}^{*}$ separating the contact wave. The HLLC numerical flux is expressed as

$$
\mathbf{F}^{\mathrm{hllc}}\left(\mathbf{Q}_{L}, \mathbf{Q}_{R}, \hat{\mathbf{n}}_{i j}\right)=\left\{\begin{array}{lll}
\mathbf{F}\left(\mathbf{Q}_{L}\right) & \text { if } \quad S_{L}>0, \\
\mathbf{F}\left(\mathbf{Q}_{L}^{*}\right) & \text { if } \quad S_{L} \leqslant 0<S_{M}, \\
\mathbf{F}\left(\mathbf{Q}_{R}^{*}\right) & \text { if } \quad S_{M} \leqslant 0 \leqslant S_{R}, \\
\mathbf{F}\left(\mathbf{Q}_{R}\right) & \text { if } \quad S_{R} \leqslant 0,
\end{array}\right.
$$

where the expressions of the acoustic waves $S_{L}, S_{M}$ and the contact wave $S_{M}$ can be found in [45]. The corresponding numerical fluxes possess good properties in resolution of shocks, contact waves and preservation of monotonicity. Finally, the semi-discrete system (6) is marched in time using an explicit three-stage third-order TVD Runge-Kutta scheme [47]

$\mathbf{Q}^{(1)}=\mathbf{Q}^{n}+\Delta t \mathbf{R}\left(\mathbf{Q}^{n}\right)$,

$\mathbf{Q}^{(2)}=\frac{3}{4} \mathbf{Q}^{n}+\frac{1}{4}\left[\mathbf{Q}^{(1)}+\Delta t \mathbf{R}\left(\mathbf{Q}^{(1)}\right)\right]$,

$\mathbf{Q}^{n+1}=\frac{1}{3} \mathbf{Q}^{n}+\frac{2}{3}\left[\mathbf{Q}^{(2)}+\frac{2}{3} \Delta t \mathbf{R}\left(\mathbf{Q}^{(2)}\right)\right]$,

where subscript $i$ was dropped for sake of clarity. Local time stepping acceleration is employed for steady state flow solution.

\section{Spatial discretization methods}

From a practical point of view, the computation of the numerical fluxes (10) requires the reconstruction of the vector of the primitive variables $\boldsymbol{q}=[\rho, u, v, p]^{\mathrm{T}}$ at both right- and left-side of the cell faces. For first-order spatial accuracy, a constant piecewise cell distribution is assumed. The Riemann states are simply taken from the cell centroids. In order to construct high-order schemes, $\boldsymbol{q}_{L}$ and $\boldsymbol{q}_{R}$ are interpolated from the cell centroids using Taylor series expansions. A simple algorithm based on the use of a least squares approach for the computations of the cell gradients is presented in Section 3.1. The resulting second-order discretization scheme (namely the LS2 scheme) will serve as a reference scheme for comparison with second- and higher-order schemes constructed using the moving Kriging (MK) shape functions described in Section 3.2. The correlation functions and a brief discussion about the construction of the domain of influence are presented in Sections 3.3 and 3.4 respectively.

\subsection{Least squares linear reconstruction}

Second-order spatial accuracy leads to the following piecewise linear component wise reconstruction of any primitive variables $q$ in the vicinity of cell centroid $\boldsymbol{x}_{0}$

$q(\boldsymbol{x})=q\left(\boldsymbol{x}_{0}\right)+\nabla q_{0}\left(\boldsymbol{x}-\boldsymbol{x}_{0}\right)$,

where $\nabla q_{0}$ is the gradient vector of the unknowns which is assumed to be constant for each cell $C_{i}$. Wang and Liu [31] computed the cell gradients by means of the following least squares fitting

$$
\left[\begin{array}{l}
q_{x} \\
q_{y}
\end{array}\right]=\frac{1}{\Delta}\left[\begin{array}{cc}
I_{y y} & -I_{x y} \\
-I_{x y} & I_{x x}
\end{array}\right]\left[\begin{array}{c}
\sum_{j=1}^{N_{f}}\left(q_{j}-q_{0}\right)\left(x_{j}-x_{0}\right) \\
\sum_{j=1}^{N_{f}}\left(q_{j}-q_{0}\right)\left(y_{j}-y_{0}\right)
\end{array}\right],
$$

where

$\Delta=I_{x x} I_{y y}-I_{x y}^{2}$ and

$$
\begin{aligned}
& I_{x x}=\sum_{j}^{N_{f}}\left(x_{j}-x_{0}\right)^{2}, \\
& I_{x y}=\sum_{j}^{N_{f}}\left(x_{j}-x_{0}\right)\left(y_{j}-y_{0}\right), \\
& I_{y y}=\sum_{j}^{N_{f}}\left(y_{j}-y_{0}\right)^{2} .
\end{aligned}
$$

This method has the advantage to use directly the unknowns at cellcentroids without depreciating the formal second-accuracy of the numerical scheme. The corresponding reconstruction stencil is simply build using the first layer of neighbors to the cell center control volume (e.g. $N_{f}=3$ for triangular elements). Previous numerical experiments have shown that the results of the LS2 scheme are comparable to those obtained from the high-resolution procedure proposed by Jawahar and Kamath [48] where the gradients of cell $C_{i}$ are computed using the area-weighted average of the corresponding face gradients. However, the present scheme is more flexible to implement and it does not require some nodal averaging procedure to compute the flow gradients.

\subsection{Higher-order discretization based on Moving Kriging shape functions}

High-order reconstructions of $\boldsymbol{q}_{L}$ and $\boldsymbol{q}_{R}$ can simply be derived, in the framework of finite volume methods, from the following expansion in the vicinity of $\boldsymbol{x}_{0}$

$\left.q(x, y) \approx \sum_{n=1}^{N} \frac{1}{n !}\left[\left(x-x_{0}\right) \frac{\partial}{\partial x}+\left(y-y_{0}\right) \frac{\partial}{\partial y}\right]^{(n)} q\left(x_{0}, y_{0}\right)\right)$.

For instance, the cubic Taylor polynomial approximation of the solution reads

$$
\begin{aligned}
q(x, y) \approx & q\left(x_{0}, y_{0}\right)+\left.\frac{\partial q}{\partial x}\right|_{\boldsymbol{x}_{0}}\left(x-x_{0}\right)+\left.\frac{\partial q}{\partial x}\right|_{\boldsymbol{x}_{0}}\left(y-y_{0}\right)+\left.\frac{1}{2} \frac{\partial^{2} q}{\partial x^{2}}\right|_{\boldsymbol{x}_{0}}\left(x-x_{0}\right)^{2} \\
& +\left.\frac{\partial^{2} q}{\partial x \partial y}\right|_{\boldsymbol{x}_{0}}\left(x-x_{0}\right)\left(y-y_{0}\right)+\left.\frac{1}{2} \frac{\partial^{2} q}{\partial y^{2}}\right|_{\boldsymbol{x}_{0}}\left(y-y_{0}\right)^{2} \\
& +\left.\frac{1}{6} \frac{\partial^{3} q}{\partial x^{3}}\right|_{\boldsymbol{x}_{0}}\left(x-x_{0}\right)^{3}+\left.\frac{1}{2} \frac{\partial^{3} q}{\partial x^{2} \partial y}\right|_{\boldsymbol{x}_{0}}\left(x-x_{0}\right)^{2}\left(y-y_{0}\right) \\
& +\left.\frac{1}{2} \frac{\partial^{3} q}{\partial x \partial y^{2}}\right|_{\boldsymbol{x}_{0}}\left(x-x_{0}\right)\left(y-y_{0}\right)^{2}+\left.\frac{1}{6} \frac{\partial^{3} q}{\partial y^{3}}\right|_{\boldsymbol{x}_{0}}\left(y-y_{0}\right)^{3}
\end{aligned}
$$

For unsteady applications, it is necessary to introduce additional terms in (17) and (18) to enforce the conservation of the mean $[4,23,49]$.

The primary objective of this study is to compute the successive derivatives at cell centroids involved in (17) by means of the moving Kriging (MK) interpolation technique for scattered data [33]. Let $\Omega_{\boldsymbol{x}}$ a compact support containing $n$ grid nodes around the node of interest $\boldsymbol{x}_{0}$. We first consider the estimated value $q^{h}$ of the function $q(\boldsymbol{x})$ at point $\boldsymbol{x}_{0}$

$q\left(\boldsymbol{x}_{0}\right) \approx q^{h}\left(\boldsymbol{x}, \boldsymbol{x}_{0}\right)=\sum_{i=1}^{n} \lambda_{i} q\left(\boldsymbol{x}_{i}\right)=\boldsymbol{\Phi}^{\mathrm{MK}}(\boldsymbol{x}) \boldsymbol{q}_{\Omega_{x}}$,

where $\boldsymbol{q}_{\Omega_{x}} \in \mathbb{R}^{n}$ contains the values at support points and the weight $\lambda_{i}$, defining the shape of the reconstructed interpolating function around node $\boldsymbol{x}_{0}$, is a function of $\boldsymbol{x}$. The shape function matrix $\boldsymbol{\Phi}^{\mathrm{MK}}(\boldsymbol{x}) \in \mathbb{R}^{1 \times n}$ is decomposed based on a regression representation plus local departures $[34,36,40]$

$\boldsymbol{\Phi}^{\mathrm{MK}}(\boldsymbol{x})=p^{\mathrm{T}}(\boldsymbol{x}) \boldsymbol{A}+\boldsymbol{r}^{\mathrm{T}}(\boldsymbol{x}) \boldsymbol{B}, \quad \forall \boldsymbol{x} \in \Omega_{\chi}$, 
where $\boldsymbol{p}^{\mathrm{T}}(\boldsymbol{x})=\left[1, x, y, x y, x^{2}, y^{2}, \ldots\right]$ is the row vector of the $m$ dimensional polynomial basis. For two-dimensional problems, the reconstructions defined by Eqs. (13) and (18) imply that $m=3$ and $m=10$ respectively. The matrix $\boldsymbol{r}^{\mathrm{T}}(\boldsymbol{x}) \in \mathbb{R}^{1 \times m}$ of correlation between the nodes of the stencil and $\boldsymbol{x}$ is defined by

$r^{\mathrm{T}}(\boldsymbol{x})=\left[C\left(\boldsymbol{x}_{1}, \boldsymbol{x}\right), \ldots, C\left(\boldsymbol{x}_{n}, \boldsymbol{x}\right)\right]$,

where $C\left(\boldsymbol{x}_{i}, \boldsymbol{x}\right)$ denotes the correlation function of the MK interpolation.

The coefficients of matrices $\boldsymbol{A} \in \mathbb{R}^{m \times m}$ and $\boldsymbol{B} \in \mathbb{R}^{m \times m}$ in (20) are determined by minimizing the mean square of the estimation error

$$
\begin{aligned}
\mathbb{E}\left[q\left(\boldsymbol{x}_{0}\right)-q^{h}\left(\boldsymbol{x}, \boldsymbol{x}_{0}\right)\right]^{2}= & \mathbb{E}\left[q\left(\boldsymbol{x}_{0}\right)\right]^{2}-\sum_{i=1}^{n} 2 \lambda_{i} \mathbb{E}\left[q\left(\boldsymbol{x}_{0}\right) q\left(\boldsymbol{x}_{i}\right)\right] \\
& +\sum_{i=1}^{n} \sum_{j=1}^{n} \lambda_{i} \lambda_{j} \mathbb{E}\left[q\left(\boldsymbol{x}_{i}\right) q\left(\boldsymbol{x}_{j}\right)\right]
\end{aligned}
$$

Moreover, the Kriging weights $\lambda_{1}, \ldots, \lambda_{n}$ must satisfy the no-bias property of the estimator which means that the expected values of $q^{h}\left(\boldsymbol{x}, \boldsymbol{x}_{0}\right)$ and $q\left(\boldsymbol{x}_{0}\right)$ are equal. The resulting linear optimization problem, which is expressed using the Lagrange function $L\left(\lambda_{i}, \mu_{k}\right)$, is characterized by a linear system of $(n+m)$ equations with respect to the unknown $\lambda \in \mathbb{R}^{m}$ and the Lagrange multipliers $\boldsymbol{\mu} \in \mathbb{R}^{\mathrm{m}}$ relative to $m$ th linear constraints

$\sum_{j=1}^{n} \lambda_{j} \mathbb{E}\left[q\left(\boldsymbol{x}_{i}\right) q\left(\boldsymbol{x}_{j}\right)\right]+\sum_{k=1}^{m} \mu_{k} p_{k}\left(\boldsymbol{x}_{j}\right)=\mathbb{E}\left[q\left(\boldsymbol{x}_{0}\right) q\left(\boldsymbol{x}_{i}\right)\right], \quad 1 \leqslant i \leqslant n$,

$\sum_{j=1}^{n} \lambda_{j} p_{k}\left(\boldsymbol{x}_{j}\right)=p_{k}\left(\boldsymbol{x}_{0}\right), \quad 1 \leqslant k \leqslant m$

where $p_{k}\left(\boldsymbol{x}_{i}\right)$ denote the monomials of the polynomial basis.

The Kriging system defined by Eqs. (23) and (24) can be written in matrix form as

$\boldsymbol{C} \lambda+\boldsymbol{P} \boldsymbol{\mu}=\boldsymbol{r}\left(\boldsymbol{x}_{0}\right)$,

$\boldsymbol{P}^{\mathrm{T}} \lambda=\boldsymbol{p}\left(\boldsymbol{x}_{0}\right)$,

where the covariance matrix $\boldsymbol{C} \in \mathbb{R}^{n \times n}$ is defined as

$\boldsymbol{C}=\left[\begin{array}{llll}1 & C\left(\boldsymbol{x}_{1}, \boldsymbol{x}_{2}\right) & \cdots & C\left(\boldsymbol{x}_{1}, \boldsymbol{x}_{n}\right) \\ C\left(\boldsymbol{x}_{2}, \boldsymbol{x}_{1}\right) & 1 & \cdots & C\left(\boldsymbol{x}_{2}, \boldsymbol{x}_{n}\right) \\ \vdots & \vdots & \ddots & \vdots \\ C\left(\boldsymbol{x}_{n}, \boldsymbol{x}_{1}\right) & C\left(\boldsymbol{x}_{n}, \boldsymbol{x}_{2}\right) & \cdots & 1\end{array}\right]$

and $\mid \boldsymbol{P}=\left[\boldsymbol{p}\left(\boldsymbol{x}_{1}\right) \boldsymbol{p}\left(\boldsymbol{x}_{2}\right) \cdots \boldsymbol{p}\left(\boldsymbol{x}_{n}\right)\right] \in \mathbb{R}^{m \times n}$ contains the values of the polynomial basis at all nodes of the stencil.

Substituting the weights $\lambda$ solved from (25) in Eq. (19) and expressing the matrix of shape functions $\boldsymbol{\Phi}^{\mathrm{MK}}(\boldsymbol{x})$, we obtain the expressions of the matrices $\boldsymbol{A}$ and $\boldsymbol{B}$ introduced in (20)

$\boldsymbol{A}=\left(\boldsymbol{P}^{\mathrm{T}} \boldsymbol{C}^{-1} \boldsymbol{P}\right)^{-1} \boldsymbol{P}^{\mathrm{T}} \boldsymbol{C}^{-1}$,

$\boldsymbol{B}=\boldsymbol{C}^{-1}(\boldsymbol{I}-\boldsymbol{P A})$.

It is possible to demonstrate that Kriging shape function possesses some useful mathematical properties such as the Kronecker delta property and the partition unity property [36]. Furthermore, any function encompassed in the basis can be reproduced exactly.

Finally, the computation of the successive derivatives of the MK shape functions can be readily obtained as

$\boldsymbol{\Phi}_{, x}^{\mathrm{MK}}(\boldsymbol{x})=\boldsymbol{p}_{, x}^{\mathrm{T}}(\boldsymbol{x}) \boldsymbol{A}+\boldsymbol{r}_{, x}^{\mathrm{T}}(\boldsymbol{x}) \boldsymbol{B}$,

$\boldsymbol{\Phi}_{, x y}^{\mathrm{MK}}(\boldsymbol{x})=\boldsymbol{p}_{x y}^{\mathrm{T}}(\boldsymbol{x}) \boldsymbol{A}+\boldsymbol{r}_{, x y}^{\mathrm{T}}(\boldsymbol{x}) \boldsymbol{B}$.

We see from (29) that all derivatives can be computed exactly very easily since we only have to deal with the derivatives of the polynomial basis and the model of covariance function. This is in contrast with the MLS approach where the high derivatives are often evaluated by means of the diffuse approximation approach $[50,20,21]$ in order to diminish coding efforts. Once the partial derivatives of the MK shape functions are evaluated, the high-order reconstruction step (17) can applied using the successive derivatives of the solution at cell centroids

$q_{, x}\left(\boldsymbol{x}_{0}\right) \approx \boldsymbol{\Phi}_{, x}^{\mathrm{MK}}\left(\boldsymbol{x}_{0}\right) q_{\Omega_{x}}$
$q_{, x y}\left(\boldsymbol{x}_{0}\right) \approx \boldsymbol{\Phi}_{, x y}^{\mathrm{MK}}\left(\boldsymbol{x}_{0}\right) q_{\Omega_{x}}$

\subsection{Correlation models}

The performances of the MK shape functions in terms of support compactness, robustness and accuracy are closely related to the choice of the correlation function. Various correlation models are employed in the field of element-free Galerkin methods for structural mechanics $[36,29,40]$. The first correlation function which be used in this work is the Gaussian correlation function $[27,36]$

$C\left(\boldsymbol{x}_{i}, \boldsymbol{x}_{j}\right)=\mathrm{e}^{-\left(\theta_{g} d_{i j} / d_{\max }\right)^{2}}$,

where $d_{i j}$ denotes the Euclidian distance between centroids of cells $i$ and $j, d_{\max }$ denotes the largest distance between the centroid of the control volume and any grid nodes of the compact support, and $\theta_{g}>0$ is the correlation coefficient which have to be chosen based on numerical experiments.

We also consider the quartic spline (QS) function [41] as a possible choice for the correlation function

$$
C\left(\boldsymbol{x}_{i}, \boldsymbol{x}_{j}\right)=1-6\left(\theta_{q s} d_{i j} / d_{\max }\right)^{2}+8\left(\theta_{q s} d_{i j} / d_{\max }\right)^{3}-3\left(\theta_{q s} d_{i j} / d_{\max }\right)^{4},
$$

where $\theta_{q s}$ denotes the corresponding correlation coefficient.

As an illustration purpose, we plot on Fig. 1 the MK interpolation obtained from the Gaussian and quartic spline correlation functions. The support has seven nodes which are equally distributed. A linear $p=1$ basis was considered. As expected, due to the Kronecker delta property, the MK approximation passes through all the nodal values of the support. Both correlation functions present a high sensitivity level to the correlation parameter. For instance, $\theta_{g}=0.7$ gives to a smooth solution around node three but it generates excessive oscillations near the boundary of the support (Fig. 1a). On the contrary, the solution obtained for high value of the correlation coefficient, say $\theta_{g}=0.7$, exhibits an irregular shape with a narrow peak around node three. Moreover, extra oscillations are still visible. A similar behavior can be observed on the plots of the shape function obtained using the quartic spline correlation (Fig. 1b).

It is interesting to note on this example that optimal values of the correlation parameters (e.g. $\theta_{g}=1.4$ and $\theta_{q s}=1$ ) deduced from the analysis on a regular support (Fig. 1) are still applicable for an irregular distribution of the support nodes of the discretization stencil (Fig. 2). However, it is obvious that the best choice correlation parameters is strongly dependent to the solution and to the size of the support. As a consequence it is not possible to design a problem-independent law in order to obtain an optimal value of the correlation parameters. Nevertheless, a judicious choice of the correlation function will give accurate results for a large range of possible values of the correlation parameters.

\subsection{Domain of influence and monotonicity enforcement}

The computation of the shape function and its derivatives at the cell centroids requires the knowledge of surrounding nodes forming a compact support (namely the reconstruction stencil). The 
(a) Gaussian correlation

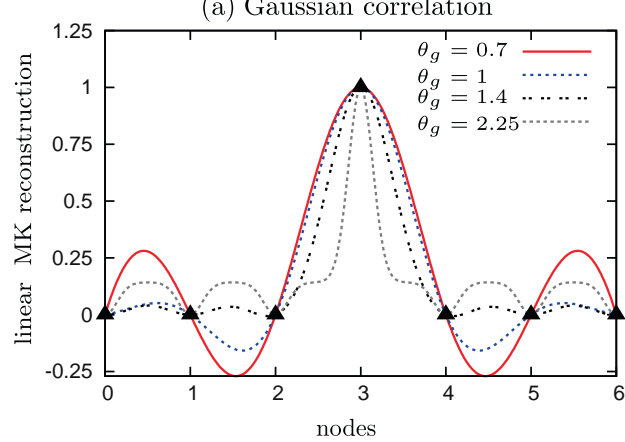

(b) Quartic spline correlation

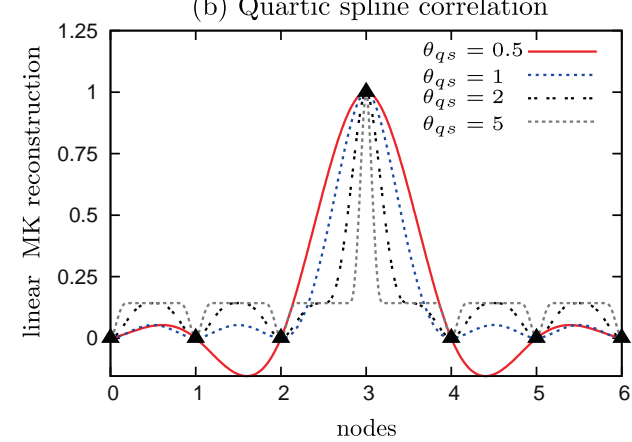

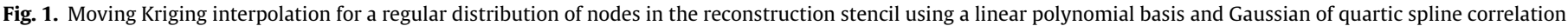
function.

(a) Gaussian correlation

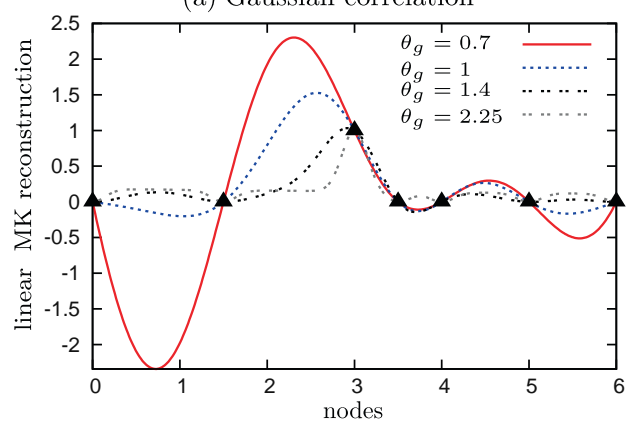

(b) Quartic spline correlation

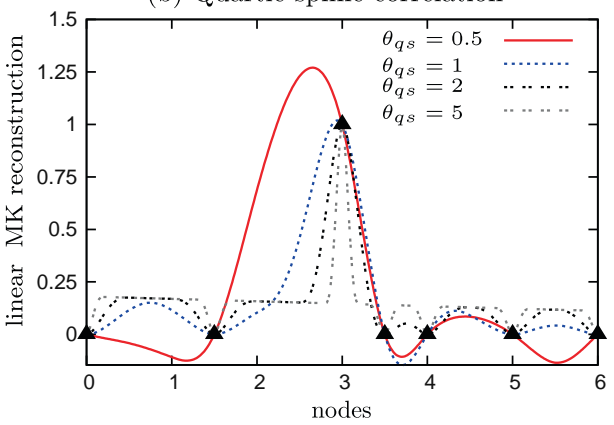

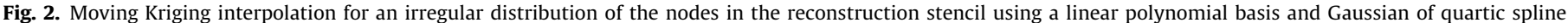
correlation function on an irregular grid.

construction of the stencil is of crucial importance in the context of high-order finite volume methods on unstructured grids $[23,11,51]$. It must be constructed to avoid ill-conditioned covariance and polynomial matrices. Furthermore, the number of control volumes inside the reconstruction stencil must be a compromise between affordable computational cost and solution accuracy. In this work, the local stencil used to compute the convective fluxes is constructed by adding neighbor elements sharing a face with cells belonging to the previous layer (Fig. 3). The minimum size of the stencil is dictated by $(p+1)(p+2) / 2$ grid nodes for a $p$ th order polynomial basis $[23,11]$. However, the size of the supporting nodes $N_{s}$ may be increased in practice to enforce the robustness of the numerical method. In this study, we will paid a particular attention to the influence of both the size of the stencil and the value of the correlation parameter on the formal accuracy of the high-order moving Kriging reconstruction scheme. Viscous computations require the evaluation of viscous fluxes at the quadrature

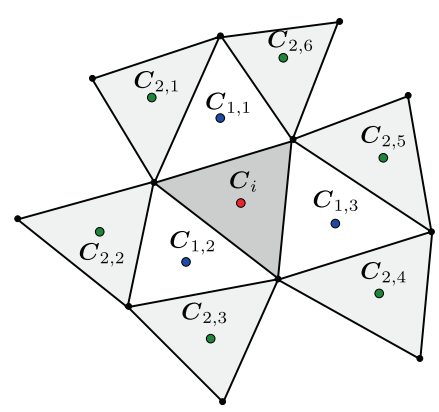

Fig. 3. Typical moving Kriging reconstruction stencil with $N_{s}=10$ control volumes employed for constructing a third-order accurate MK scheme. points of all the faces of the control volume. Thus, it is required to compute the flow variables at these points. These are easily computed as:

$v_{q}=\sum_{j=1}^{n_{q}} v_{j} \Phi_{j}\left(\boldsymbol{x}_{q}\right), \quad T_{q}=\sum_{j=1}^{n_{q}} T_{j} \Phi_{j}\left(\boldsymbol{x}_{q}\right)$,

$\nabla v_{q}=\sum_{j=1}^{n_{q}} v_{j} \otimes \nabla \Phi_{j}\left(\boldsymbol{x}_{q}\right), \quad \nabla T_{q}=\sum_{j=1}^{n_{q}} T_{j} \nabla \Phi_{j}\left(\boldsymbol{x}_{q}\right)$.

The corresponding viscous MK stencil is simply obtained by merging all of the MK stencils of the first neighbors of the cell of interest. From a practical point of view, the construction of the discretization stencil and the computation of the MK shape function derivatives are done as a preprocessing step prior to the iterative procedure.

In order to prevent nonphysical oscillations in the solution for non-smooth flows, we use a shock-capturing procedure where slope-limiters are applied on both the linear part and the high-order terms in the reconstruction step (17). To this end, the multidimensional limiting procedure based on a three-gradient limiter developed by Jawahar and Kamath [48] for second-order discretization on unstructured triangular grids is applies to the present high-order method. It must be noted that more advanced limiting techniques can be employed in the context of reproducing kernel finite volume methods such as selective limiting based on MLS sensor which can be regarded as a high-pass filter to detect flow discontinuities [52].

\section{Numerical tests for inviscid flows}

In this section, the accuracy and robustness of the high-order FV-MK algorithm are studied for steady inviscid transonic flow problems with smooth or discontinuous solutions. 


\subsection{Ringleb flow}

First, the accuracy of the present FV-MK solver is investigated for the case of the Ringleb flow problem. This configuration, which is widely used for accuracy assessment of high-order unstructured methods [53-56,24], corresponds to a smooth flow in a transonic curved channel. Numerical results are compared with the exact solution obtained from the hodograph method for the steady Euler equations [57]. The flow is described by means of the streamline constant $k$ and the total velocity q. Fig. 4 shows the isolines of the Mach number obtained from the exact solution for the case of left and right streamline constants equal to $k_{\text {left }}=0.55$ and $k_{\text {right }}=1.05$ respectively. The inflow boundary condition is defined by $q_{\text {inflow }}=0.35$. The transonic outflow boundary corresponds to $y=0$. A small supersonic region is clearly visible near the bottom left streamline boundary with a maximum Mach number equal to 1.2 (Fig. 4). As a consequence, the outflow exhibits both subsonic and supersonic conditions. The accuracy study is performed using a sequence of three computational grids with irregular triangular cells as depicted in Fig. 4. The corresponding number of control volumes for the coarse, medium and fine mesh are 131, 566 and 2328 respectively.

In the following, the convergence rates are monitored by means of a log-log plot of the $L_{1}$ - and $L_{2}$-norm of the error in the entropy production as a function of the number of control volumes of the computational grid. First, we compare the solution errors obtained using the second-order least squares (LS2) scheme and the moving Kriging reconstruction using Gaussian (MK2-G) or quartic-spline (MK2-QS) correlation functions. The correlation parameter is $\theta_{g}=\theta_{q s}=1.5$. We keep the same size $N_{\mathrm{s}}=4$ of the discretization support for both LS2 and MK2. We can observed on Fig. 5 that all schemes present a correct asymptotic rates of convergence. The differences between the Gaussian and quartic spline correlation functions are undistinguishable. However, it is clearly visible that MK-based schemes give the smallest error level which means that the approximation of the first derivatives is more accurate than for the case of the least squares approach. This remark is confirmed by the examination of Fig. 6 which presents a comparison between LS2 and MK2-G on the discrete error field in density defined as $\operatorname{err}\left(\rho_{i}\right)=\left|\rho_{i}-\rho_{\text {exact }}\left(x_{i}, y_{i}\right)\right|$. The regions dominated by high errors are mainly located in the transonic flow zone close to the left boundary (Fig. 6a). We notice on Fig. 6b that the maximum error can be reduced by almost a factor two when MK shape functions are employed in the reconstruction step instead of the LS2 scheme.

Table 1 summarizes the error norms and the order of convergence for second-order accurate solution algorithms presented in Fig. 5. To this end, the experimental order of convergence is evaluated by comparison between the solution computed on two grids with characteristic mesh size $h_{a}$ and $h_{b}$ as follows

$\operatorname{Ord}_{a b}=\frac{\log \left(\mathrm{err}_{\mathrm{a}}\right)-\log \left(\mathrm{err}_{\mathrm{b}}\right)}{\log h_{a}-\log h_{b}}$

where $\operatorname{err}_{\text {a.b.c }}$ denotes either the $L_{1}$-norm or the $L_{2}$-norm of the error in the entropy production for the various computational grids of interest. We notice that the level of discretization error resulting from the MK interpolation exceeds slightly the theoretical $O\left(h^{2}\right)$ order of accuracy, especially for the coarse to medium grid level (Table 1). As expected, MK-G and MK-QS give similar results. Recall
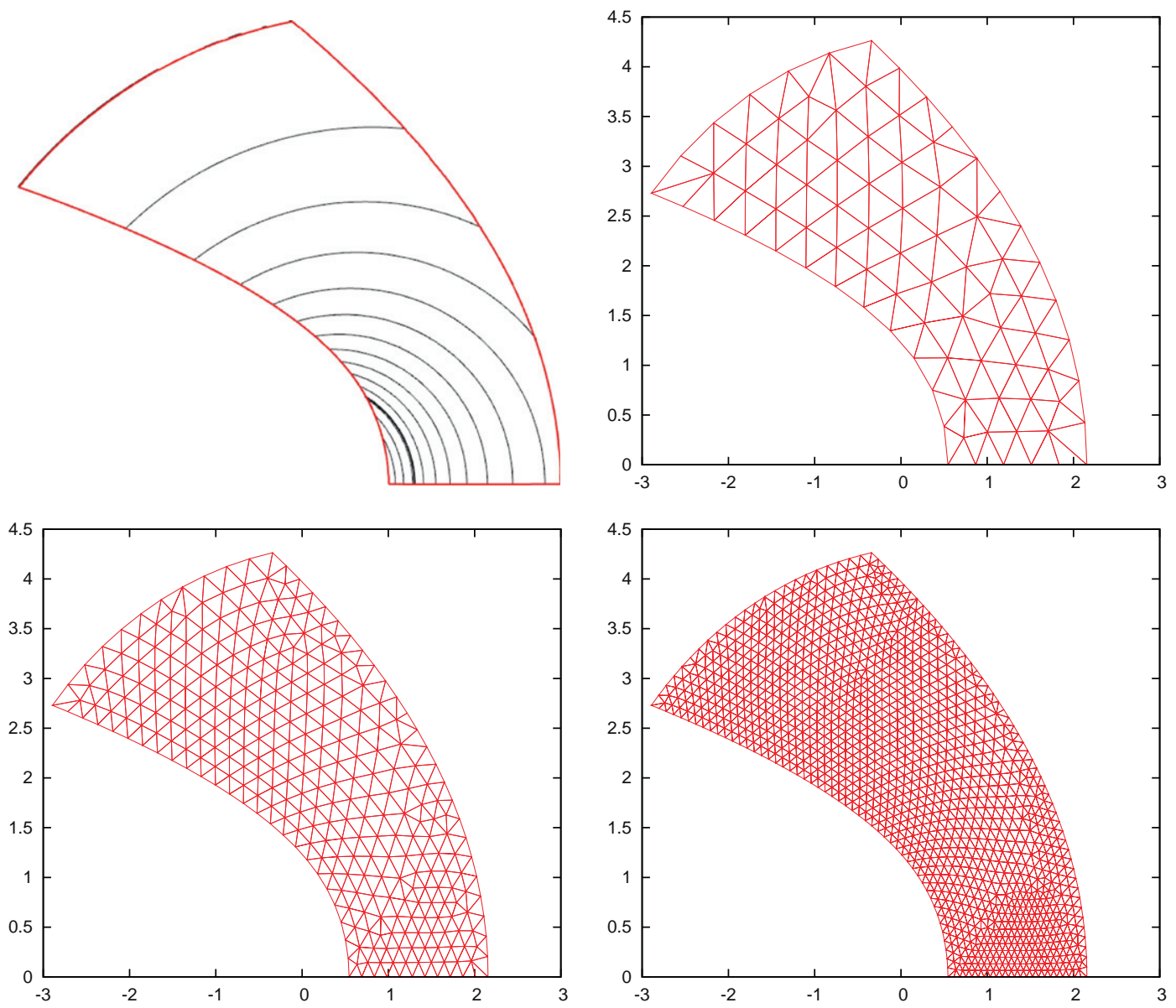

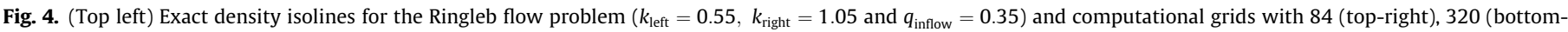
left) and 1239 elements (bottom-right). 

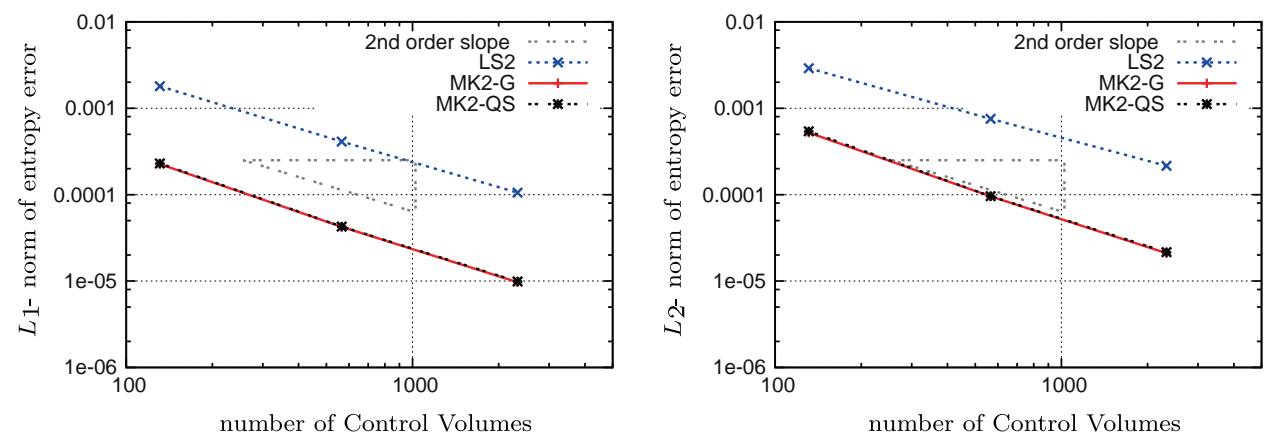

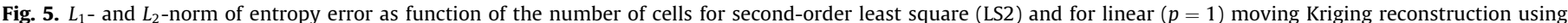
Gaussian (MK2-G) or quartic-spline (MK2-QS) correlation functions $\left(N_{s}=4, \theta_{g}=\theta_{q s}=1.5\right)$.
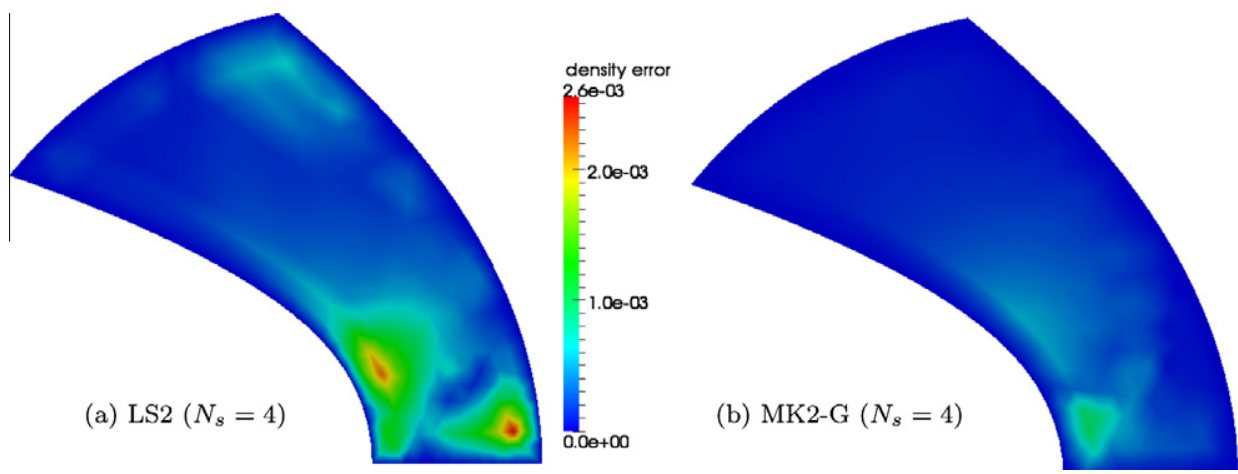

Fig. 6. Discrete error of the density computed on an unstructured grid with 320 elements for (a) LS2 scheme and (b) MK2-G scheme with $\theta_{g}=1.5$.

Table 1

$L_{1}$ and $L_{2}$ norms of error in entropy production and orders of accuracy of the FV-MK solver for the ringleb flow problem $\left(N_{s}=4, \theta_{g}=\theta_{q s}=1.5\right)$.

\begin{tabular}{|c|c|c|c|c|c|c|}
\hline Method & Mesh & Elements & $L_{1}$-error & Order & $L_{2}$-error & Order \\
\hline \multirow[t]{3}{*}{ LS2 } & $\mathrm{a}$ & 131 & $1.7991 \mathrm{E}-03$ & - & $2.9073 \mathrm{E}-03$ & - \\
\hline & $\mathrm{b}$ & 566 & $4.1116 \mathrm{E}-04$ & 2.02 & $7.5288 \mathrm{E}-04$ & 1.85 \\
\hline & c & 2328 & $1.0534 \mathrm{E}-04$ & 1.93 & $2.1503 \mathrm{E}-04$ & 1.77 \\
\hline \multirow[t]{3}{*}{ MK2-G } & $\mathrm{a}$ & 131 & $2.2595 \mathrm{E}-04$ & - & $5.2184 \mathrm{E}-04$ & - \\
\hline & $\mathrm{b}$ & 566 & $4.2374 \mathrm{E}-05$ & 2.29 & $9.5648 \mathrm{E}-05$ & 2.32 \\
\hline & $\mathrm{c}$ & 2328 & $9.7102 \mathrm{E}-06$ & 2.08 & $2.1034 \mathrm{E}-05$ & 2.14 \\
\hline \multirow[t]{3}{*}{ MK2-QS } & $\mathrm{a}$ & 131 & $2.2966 \mathrm{E}-04$ & - & $5.3935 \mathrm{E}-04$ & - \\
\hline & $\mathrm{b}$ & 566 & $4.2762 \mathrm{E}-05$ & 2.30 & $9.6074 \mathrm{E}-04$ & 2.36 \\
\hline & $\mathrm{c}$ & 2328 & $9.9039 \mathrm{E}-06$ & 2.07 & $2.1610 \mathrm{E}-05$ & 2.11 \\
\hline
\end{tabular}

that the smallest admissible MK-stencil (e.g. $\left.N_{s}=4\right)$ is sufficient to achieve the expected second-order accuracy, meaning that no extra computational costs are required compared to the LS2 scheme.

As a global indicator representative of the solution accuracy, we perform a least squares curve fit of the $L_{2}$-norm given in Fig. 6 and in Table 1 for the three grid levels. The corresponding slopes are $1.81,2.23$ and 2.24 for LS2, MK-G and MK-QS respectively.

Now, the previous computations based on the MK schemes (namely the MK-G and MK-QS) are repeated for different combinations of the correlation parameters $\theta_{g}$ and $\theta_{q s}$ ranging from 0.01 to 10. The number of elements in the stencil is $N_{s}=4$ or $N_{s}=7$. The slopes of the $L_{1}$ - and $L_{2}$ - norm in the entropy error are presented in Fig. 7. It clearly appears that the global order of convergence is not sensitive to the correlation parameter as far values of the correlation parameter are greater than 5 . The MK-QS scheme was found to be at least second-order accurate over the whole range $[0.01,10]$ for both $N_{s}=4$ and $N_{s}=7$. On the contrary, it was not possible to obtain a solution for the MK-G scheme when the correlation parameter is below than unity. In general, higher rates of conver- gence are achieved when additional cells are added in the MKstencil. However these differences remain small as far as secondorder accurate schemes are concerned.

Next, we investigate the properties of high-order accurate MK schemes based on quadratic $(p=2)$ and cubic $(p=3)$ polynomial basis. A Gaussian correlation function was considered and the same correlation parameters $\theta_{g}=10$ was retained for the formally third- (MK3-G) and fourth-order accurate (MK4-G) schemes. The reduction of the discrete error field in density obtained using MK3-G (Fig. 8a) is clearly visible compared to MK2-G (Fig. 6). In particular, we remark that the maximum error is divided by one order of magnitude. The effect of the increase of the spatial accuracy from the third-order MK3-G scheme to the fourth-order MK4-G scheme is clearly noticeable on Fig. 8b where the discretization error is improved both in the region of strong gradients and near the inflow boundary.

The $L_{1}$ - and $L_{2}$-error norm of the entropy production are shown in Fig. 9 and in Table 2. In order to verify that the correct order of accuracy is preserved for very fine grids, we consider an additional 
(a) $L_{1}-$ norm

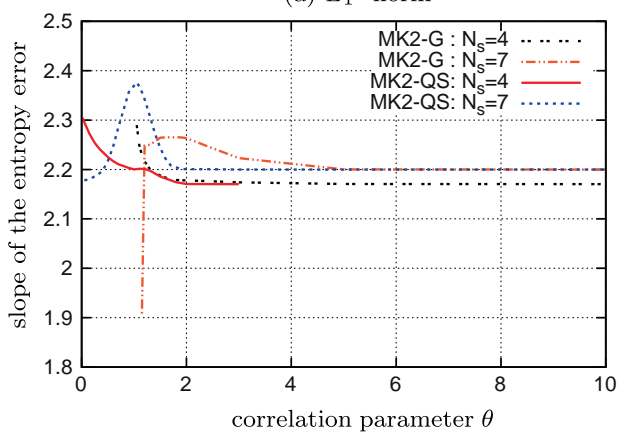

(b) $L_{2}$ - norm

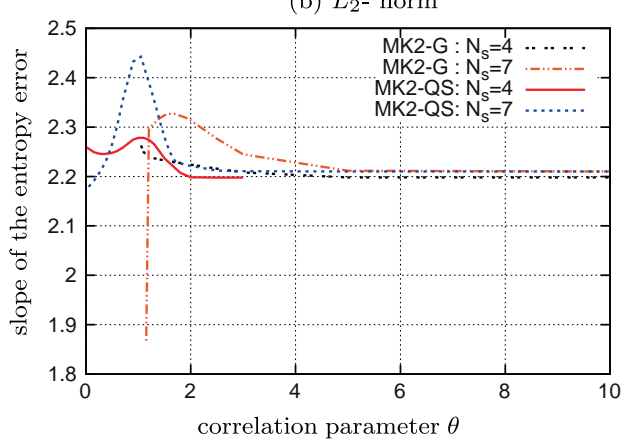

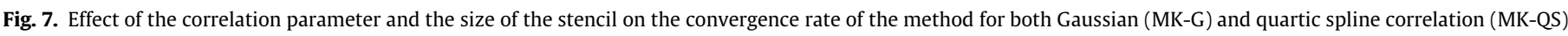
functions.
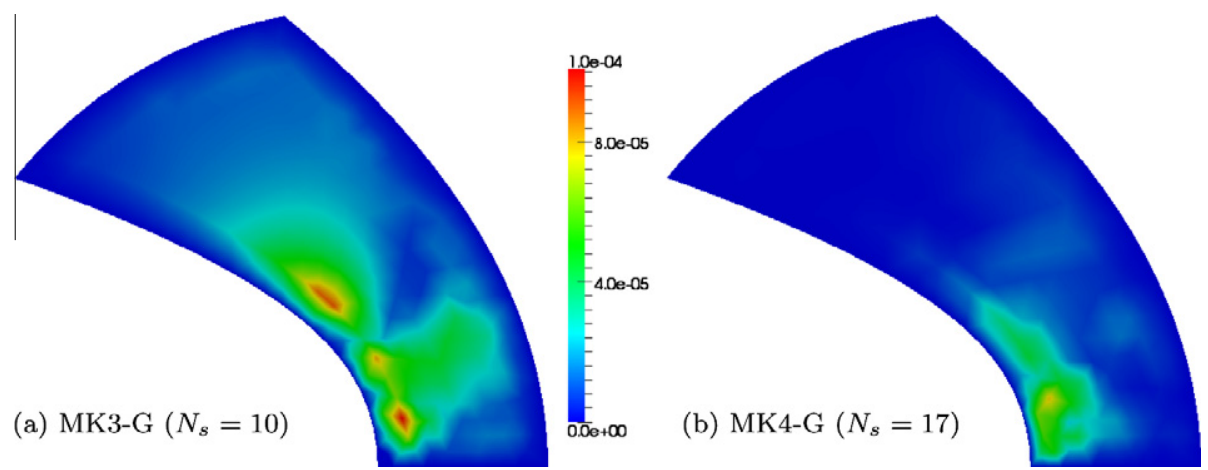

Fig. 8. Discrete error of the density computed on an unstructured grid with 320 elements for MK3-G (left) and MK4-G (right) with $\theta_{g}=10$ for both schemes.
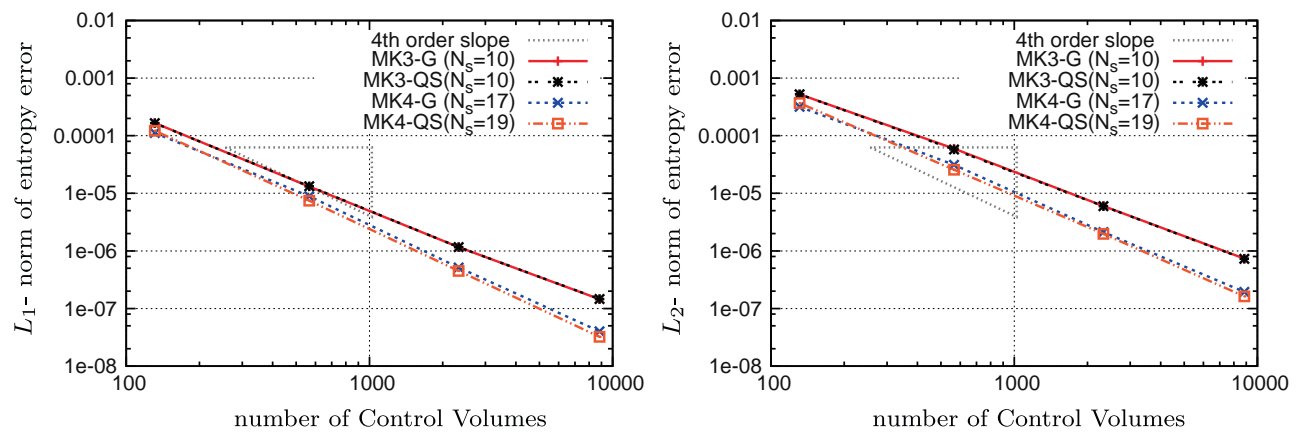

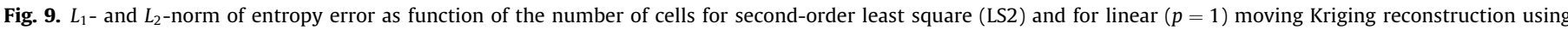
Gaussian (MK2-G) or Quartic-Spline (MK2-QS) correlation function.

unstructured mesh with 8832 cells and 4563 nodes. The size of the reconstruction stencil $N_{s}$ was chosen as small as possible, based on robustness considerations. The correlation factor is $\theta=10$ for both MK3-G and MK4-G computations. For quartic spline correlation functions, we used $\theta_{q s}=3$ and $\theta_{q s}=5$ for third- and fourth-order MK schemes respectively. The Gaussian and quartic spline correlation functions with $N_{s}=10$ give identical results for third-order MK3 schemes. Minor differences are visible for the fourth-order discretization where it seems that MK4-QS is slightly more accurate than MK4-G. However, the two approaches exhibit similar convergence rates as shown in Fig. 9.

We remark on Table 2 that the expected convergence rates of $p+1$ is recovered with the $L_{1}$-norm. This is an interesting result because it means that MK schemes are accurate using relatively small stencils, thus limiting excessive computational costs. It must also be noted that the formal fourth-order accuracy of the MK4-G scheme is depreciated for the $L_{2}$-norm in comparison with the $L_{1}$ norm (Table 2), especially for coarse grids.

Table 3 presents the influence of $N_{s}$ on the $L_{1}$ and $L_{2}$ slopes resulting from the curve fit of the convergence rates over the four computational grids for both MK3-G and MK4-G. We notice that MK3-G with $\theta_{g}=10$ is not very sensitive to $N_{s}$. On the contrary, it is necessary to use large stencils for MK4-G if we aim to recover the fourth-order formal accuracy (Table 3 ).

The density field obtained using MK4-G with $N_{s}=17$ and $\theta_{g}=10$ on the coarse grid with 131 control volumes is shown in Fig. 10a. The distribution of the density profile along the line starting from point $A$ with position $\left(x_{A}, y_{A}\right)=(-1.7,3.7)$ to point B with $\left(x_{B}, y_{B}\right)=(1.5,0)$ is presented in Fig. $10 \mathrm{~b}$ for all MK discretization orders. The improvement due to the use of higher-order representations is clearly visible in the transonic region. 
Table 2

$L_{1}$ and $L_{2}$ norms of error in entropy production and corresponding convergence rates for $p=2$ and $p=3$ MK schemes.

\begin{tabular}{|c|c|c|c|c|c|c|c|c|}
\hline Method & Mesh & Elements & $N_{s}$ & $\theta$ & $L_{1}$-error & Order & $L_{2}$-error & Order \\
\hline \multirow[t]{4}{*}{ MK3-G } & $\mathrm{a}$ & 131 & 10 & 10 & $1.6638 \mathrm{E}-04$ & - & $5.2349 \mathrm{E}-04$ & - \\
\hline & $\mathrm{b}$ & 566 & 10 & 10 & $1.3118 \mathrm{E}-05$ & 3.47 & $5.9560 \mathrm{E}-05$ & 2.97 \\
\hline & c & 2328 & 10 & 10 & $1.1670 \mathrm{E}-06$ & 3.42 & $6.0104 \mathrm{E}-06$ & 3.24 \\
\hline & d & 8832 & 10 & 10 & $1.4505 \mathrm{E}-07$ & 3.13 & $7.3522 \mathrm{E}-07$ & 3.15 \\
\hline \multirow[t]{4}{*}{ MK3-QS } & $\mathrm{a}$ & 131 & 10 & 3 & $1.6508 \mathrm{E}-04$ & - & $5.2266 \mathrm{E}-04$ & - \\
\hline & $\mathrm{b}$ & 566 & 10 & 3 & $1.3235 \mathrm{E}-05$ & 3.45 & $5.7696 \mathrm{E}-05$ & 3.01 \\
\hline & c & 2328 & 10 & 3 & $1.1638 \mathrm{E}-06$ & 3.44 & $5.9940 \mathrm{E}-06$ & 3.20 \\
\hline & $\mathrm{d}$ & 8832 & 10 & 3 & $1.4617 \mathrm{E}-07$ & 3.10 & $7.2876 \mathrm{E}-07$ & 3.16 \\
\hline \multirow[t]{4}{*}{ MK4-G } & $\mathrm{a}$ & 131 & 17 & 10 & $1.1149 \mathrm{E}-04$ & - & $3.1779 \mathrm{E}-04$ & - \\
\hline & $\mathrm{b}$ & 566 & 17 & 10 & $8.8799 \mathrm{E}-06$ & 3.46 & $3.1273 \mathrm{E}-05$ & 3.17 \\
\hline & c & 2328 & 17 & 10 & $5.1682 \mathrm{E}-07$ & 4.02 & $2.1290 \mathrm{E}-06$ & 3.80 \\
\hline & $\mathrm{d}$ & 8832 & 17 & 10 & $4.0172 \mathrm{E}-08$ & 3.83 & $1.9420 \mathrm{E}-07$ & 3.59 \\
\hline \multirow[t]{4}{*}{ MK4-QS } & $\mathrm{a}$ & 131 & 19 & 5 & $1.2164 \mathrm{E}-04$ & - & $3.6752 \mathrm{E}-04$ & - \\
\hline & $\mathrm{b}$ & 566 & 19 & 5 & $7.4405 \mathrm{E}-06$ & 3.82 & $2.5592 \mathrm{E}-05$ & 3.64 \\
\hline & c & 2328 & 19 & 5 & $4.5067 \mathrm{E}-07$ & 3.97 & $1.9795 \mathrm{E}-06$ & 3.62 \\
\hline & $\mathrm{d}$ & 8832 & 19 & 5 & $3.2331 \mathrm{E}-08$ & 3.95 & $1.6315 \mathrm{E}-07$ & 3.74 \\
\hline
\end{tabular}

Table 3

Influence of the size of the stencil $N_{s}$ on the slope of entropy error computed for MK3$\mathrm{G}$ and MK4-G using and $\theta_{g}=10$ for a sequence of four grids.

\begin{tabular}{|c|c|c|c|c|c|}
\hline \multicolumn{3}{|c|}{ MK3-G $(p=2)$} & \multicolumn{3}{|c|}{ MK4-G $(p=3)$} \\
\hline$N_{s}$ & Slope $L_{1}$ & Slope $L_{2}$ & $N_{s}$ & Slope $L_{1}$ & Slope $L_{2}$ \\
\hline 10 & 3.35 & 3.13 & 17 & 3.79 & 3.54 \\
\hline 15 & 3.46 & 3.17 & 21 & 3.96 & 3.68 \\
\hline 20 & 3.37 & 3.37 & 26 & 4.1 & 3.72 \\
\hline
\end{tabular}

\subsection{Inviscid flow around a NACA 0012 airfoil}

In this section, we investigate the performance of the high-order moving Kriging reconstruction for the case of an inviscid flow past a NACA 0012 airfoil. The unstructured triangular mesh shown in Fig. 11 has 158 nodes on the solid wall and 40 nodes on the outer boundary. The far field is situated at 25 chords from the airfoil. The corresponding number of cells in the computational domain is 6274. Characteristic boundary conditions based on the Riemann invariants are implemented in the far field. At the solid wall, we prescribe impermeability conditions for straight sided bodies [3]. Ghost states at Gauss-Legendre integration points are constructed from the interior state which is obtained using the same high-order reconstruction procedure employed for the computation of the Riemann problem for interior cells.

The first case corresponds to a subsonic flow with a free stream Mach number $M=0.63$ and an angle of attack $\alpha=2^{\circ}$. Since we solve a smooth flow over the airfoil without any discontinuities [58], slope limiters are not employed for this problem. Computa- tions are performed until the residuals fall below $10^{-12}$. The initial flow field corresponds to the free stream conditions. The contours of the Mach number are plotted in Fig. 12. We remark that the results obtained using LS2 exhibits isolines with some cups at the solid wall and around the trailing edge (Fig. 12a). This is obviously the signature of spurious entropy production. It is clearly visible that this drawback may be raised using the MK2 scheme whose Mach number isolines are smoother. Fig. 12b shows that the use of quadratic (MK2) and cubic (MK4) polynomial basis gives comparable results for the computational grid of interest.

The distribution of the pressure coefficient along the chord of the airfoil is presented in Fig. 13a for different reconstruction techniques. We notice that the flow acceleration around the leading edge is correctly predicted for both second-order least squares and moving Kriging schemes. The third-order MK reconstruction gives similar results. On the contrary, dramatic improvements in the production of spurious entropy production $\epsilon_{\text {ent }}$ are observed on the airfoil surface (Fig. 13b) when MK interpolations are employed. In particular, the maximum entropy error obtained using LS2 is $1.02 \times 10^{-2}$ and those computed from MK2 is $1.10 \times 10^{-3}$. This decrease corresponds to a reduction by about one order of magnitude. We also remark on Fig. 13b that the use of a third-order MK limits the production of spurious entropy compared to its second-order counterpart excepted near the leading edge.

The lift $\left(C_{L}\right)$ and drag $\left(C_{D}\right)$ coefficients are reported in Table 4 for all discretization orders employed in the MK reconstruction step. Here, we considered a Gaussian correlation function with a correlation parameter $\theta_{g}=20$. The size of the stencil $N_{s}$ ranges from five control volumes for MK2 up to 20 elements for MK4.
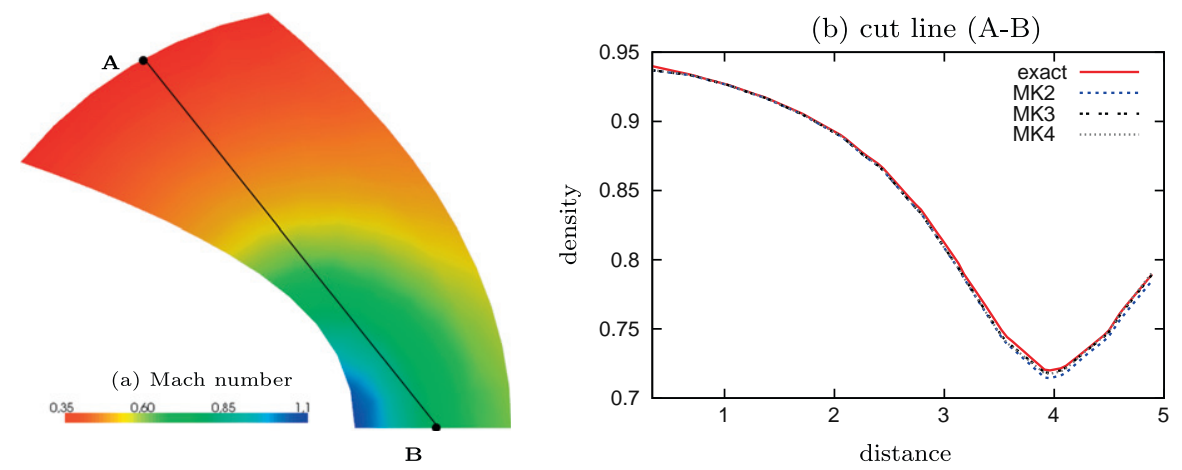

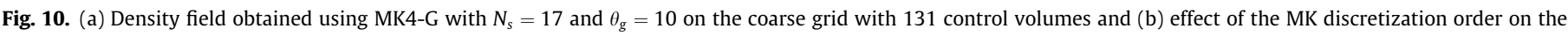
density profiles along line A-B. 


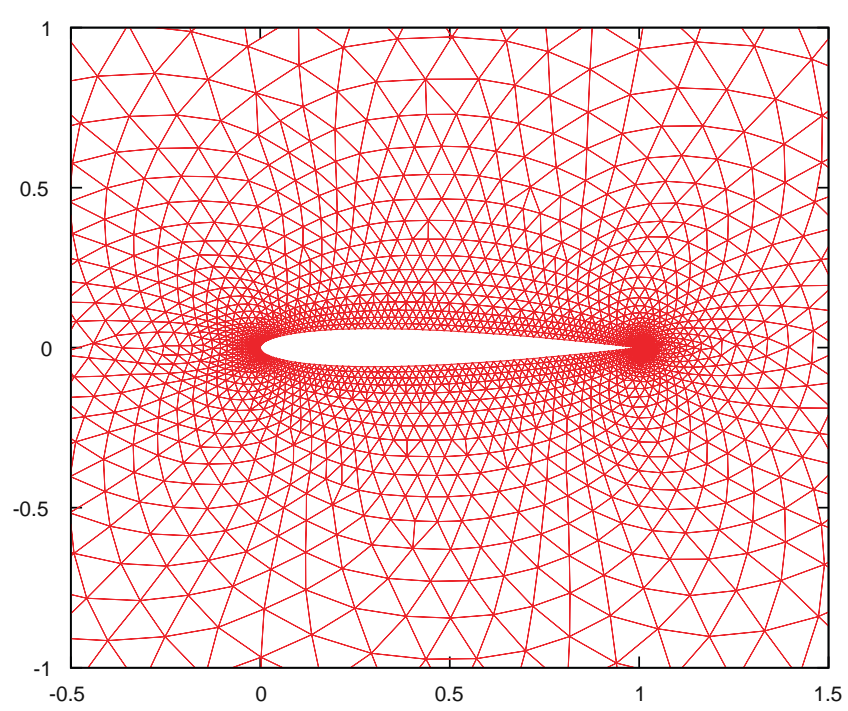

Fig. 11. Unstructured grid used for the inviscid computations past a NACA 0012 airfoil.

For comparison purposes, we have tabulated in Table 4 the results from previous studies based on a third-order DG method [3] or on a fourth-order finite volume approach combined with a piecewise polynomial reconstruction procedure [10]. The number of control volumes used in the computational grids is also reported. As expected, the use of MK reconstruction gives better prediction for both $C_{L}$ and $C_{D}$ than the LS2 scheme. We notice that increasing the discretization order results in dramatic reductions of the drag coefficient. The value of $C_{D}$ obtained for a cubic polynomial basis is close to those obtained using third-order DG method [3] but the lift coefficient is underestimated. Results obtained using a fourth-order MK discretization are similar to the values reported in [10].

Next, we consider a transonic flow condition defined by $M=0.8$ and $\alpha=1.25^{\circ}$. This configuration [58] is characterized by the presence of a weak shock on the pressure side and a strong shock on the suction side. The computational grid and numerical parameters are unchanged compared to the subsonic case. Slope-limiters are employed to avoid numerical oscillations in the vicinity of flow discontinuities. Fig. 14 shows the distribution of the pressure coefficient on the profile for different discretization orders. All schemes give similar predictions of the location of the strong shock on the upper surface. It appears on Fig. 14b that high-order reconstructions produce moderate oscillations downstream of the discontinuity which are not observed on second-order MK results.

The isolines of the Mach number obtained for a fourth-order MK scheme are presented in Fig. 15a and the corresponding distribution of entropy generation on the airfoil surface is plotted in Fig. 15b. The use of a second-order MK reconstruction reduces the entropy production compared to LS2 except locally on the shock wave and near the leading edge. On the other hand, the third-order MK discretization fails to decrease the generation of entropy. This is due to the fact that our limiting procedure, which is based on the multidimensional limiters developed by Jawahar and Kamath [48] for second-order discretization, is applied in a similar manner to both the linear and the high-order part. Less diffusive limiters can be designed using shock detector for selective limiting $[59,10,52]$ in order to recover the full high-order accuracy for smooth flows. However, we notice from Table 4 that both $C_{L}$ and $C_{D}$ obtained from a MK reconstruction are similar to the results reported in the literature using high-order discretization $[52,10]$.
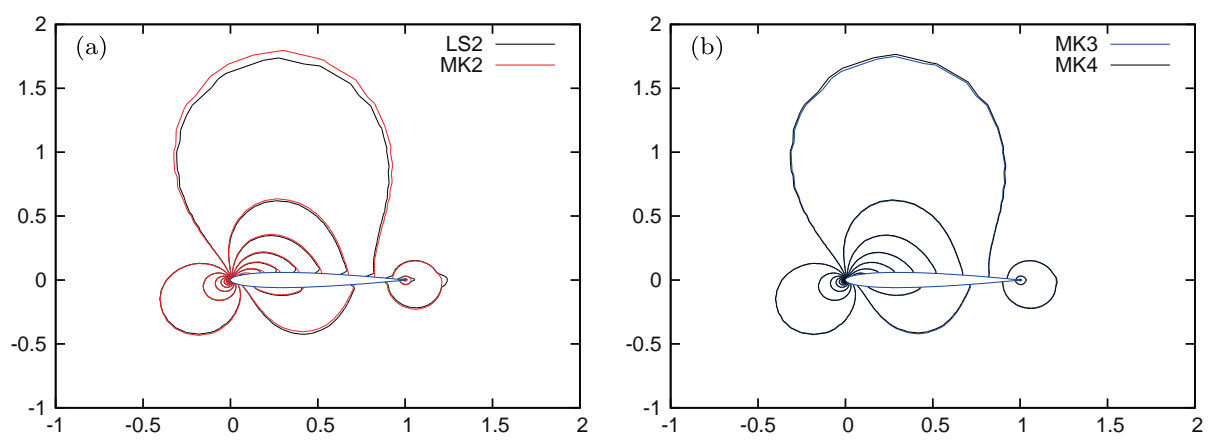

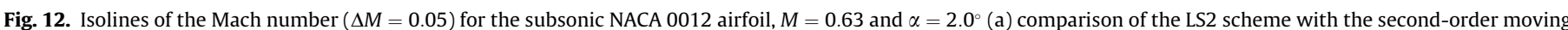
Kriging scheme (MK2) with $\theta_{g}=20$ (b) effect of the MK discretization order $p=2$ and $p=3$.
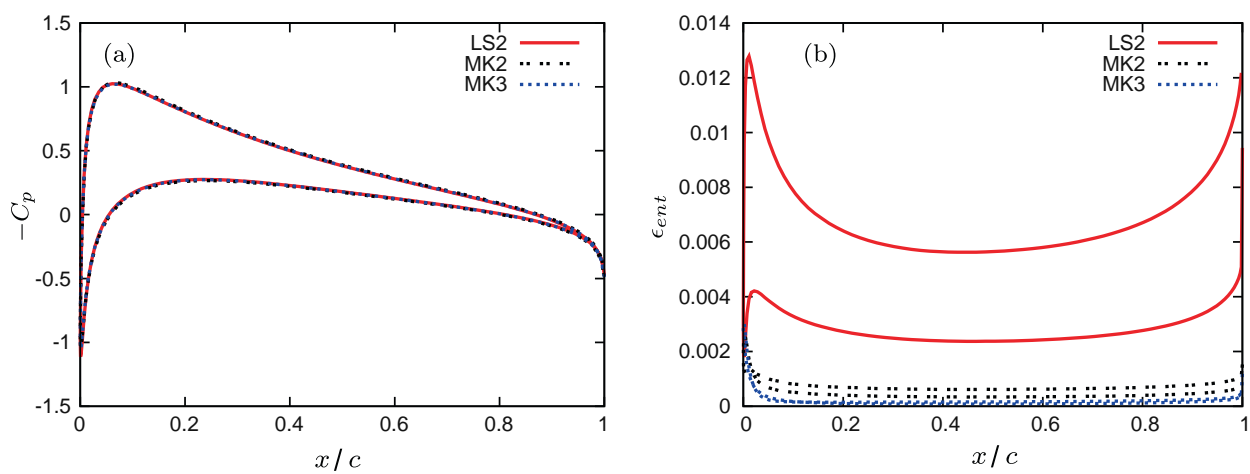

Fig. 13. Inviscid subsonic flow past NACA 0012 airfoil $M=0.63$ and $\alpha=2^{\circ}$ (a) pressure coefficient and (b) entropy production on the airfoil surface. 
Table 4

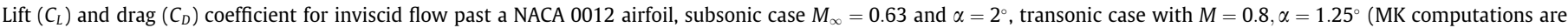
performed using a Gaussian correlation function with $\theta_{g}=20$ ).

\begin{tabular}{|c|c|c|c|c|c|c|c|}
\hline \multirow[t]{2}{*}{ Numerical scheme } & \multirow[t]{2}{*}{ Order } & \multirow[t]{2}{*}{ CVs } & \multirow[t]{2}{*}{$N_{s}$} & \multicolumn{2}{|c|}{$M_{\infty}=0.63, \alpha=2$} & \multicolumn{2}{|c|}{$M_{\infty}=0.85, \alpha=1$} \\
\hline & & & & $C_{L}$ & $C_{D}$ & $C_{L}$ & $C_{D}$ \\
\hline DG [3] & 3rd & 2960 & - & 0.333 & $1.50 \mathrm{E}-04$ & - & - \\
\hline FV $k$-exact [10] & 4 th & 9931 & 16 & 0.325 & $3.25 \mathrm{E}-04$ & 0.347 & $2.24 \mathrm{E}-02$ \\
\hline LS2 & 2nd & 6274 & 4 & 0.316 & $3.47 \mathrm{E}-03$ & 0.349 & $2.27 \mathrm{E}-02$ \\
\hline MK2-G & 2nd & 6274 & 5 & 0.330 & $3.29 \mathrm{E}-03$ & 0.339 & $2.28 \mathrm{E}-02$ \\
\hline MK3-G & $3 r d$ & 6274 & 8 & 0.318 & $1.67 \mathrm{E}-04$ & 0.346 & $2.33 \mathrm{E}-02$ \\
\hline MK4-G & 4 th & 6274 & 20 & 0.324 & $3.25 \mathrm{E}-05$ & 0.347 & $2.25 \mathrm{E}-02$ \\
\hline
\end{tabular}
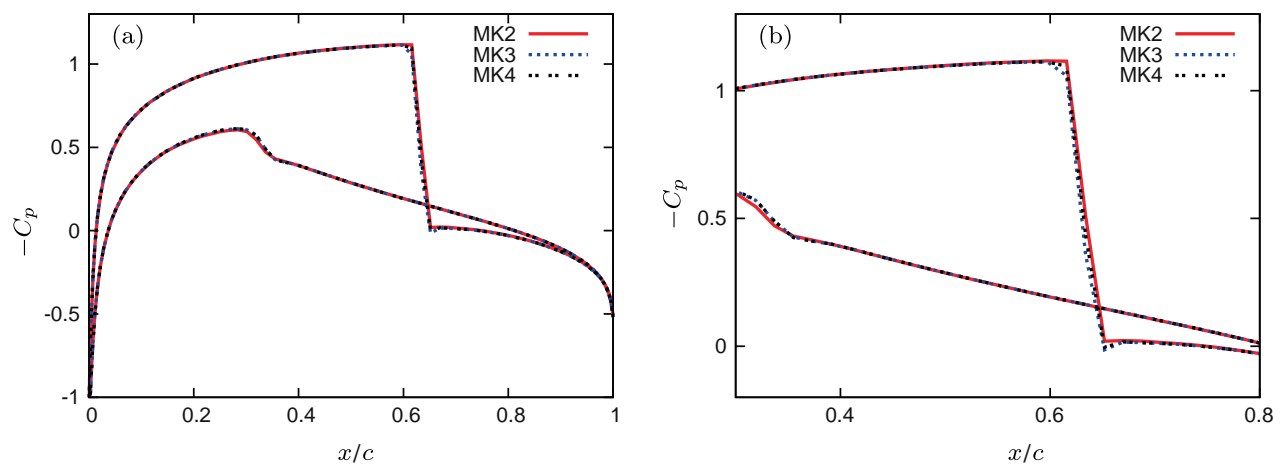

Fig. 14. Inviscid transonic flow past a NACA 0012 profile, $M=0.8, \alpha=1.25^{\circ}$. (a) Pressure coefficient distribution on the airfoil (b) closeup view around the shock wave.
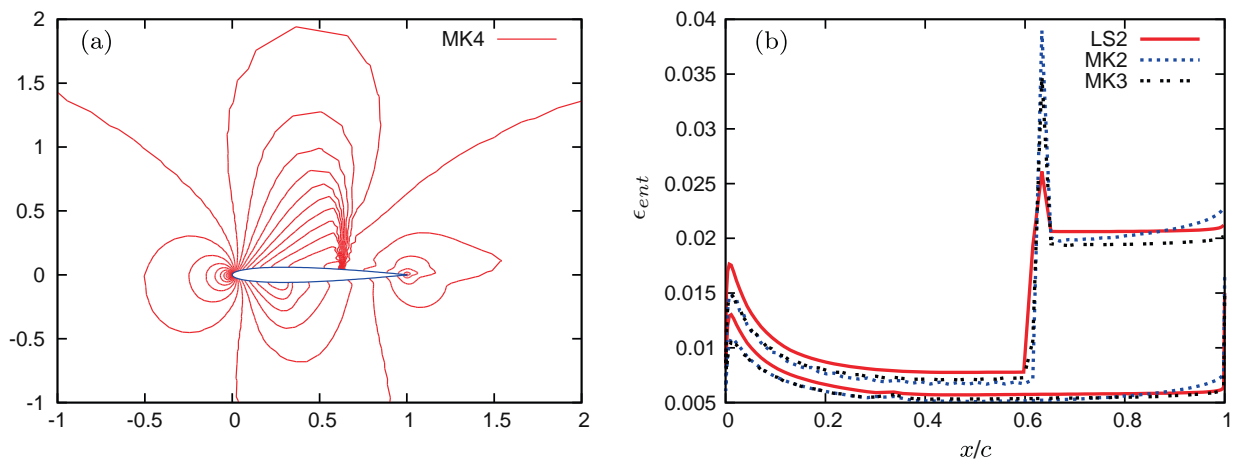

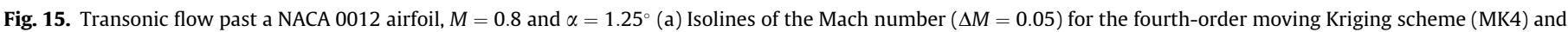

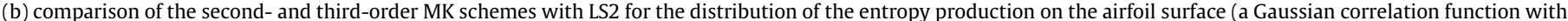
$\theta_{g}=20$ is used for all MK computations).

\section{FV-MK computations of unsteady flows}

Here, the performance of the FV-MK scheme is investigated in the context of 2D and 3D unsteady flows using either the linearized Euler equations or the Navier-Stokes equations. Third-order accurate computations are addressed by means of explicit time integration schemes with zero-mean reconstruction properties $[4,60]$.

\subsection{Acoustic pulse}

The objective of this test is to illustrate the capability of the presented MK scheme for the problem of wave propagation on arbitrary unstructured grids. To this end, the present FV-MK method, which is deployed for the solution of the linearized Euler equations, is used to propagate a Gaussian pulse with zero mean flow. The computational domain corresponds to a square extending from -100 to 100 in the two space directions. The source, located at $x_{s}=y_{s}=0$, is defined as
$S=\exp \left(-\frac{\ln (2)}{9}\left(x^{2}+y^{2}\right)\right) \times\left[\begin{array}{llll}1 & 0 & 0 & 1\end{array}\right]^{T}$

Several configurations are tested based on different values of the Gaussian correlation coefficient $\left(\theta_{g}=10,15\right.$ and 20). Time integration is performed using an explicit low-dissipation low-dispersion Runge-Kutta scheme [61]. The results are stopped at $t=50$ before the wave reaches the domain boundaries to avoid the eventually spurious waves. Fig. 16 shows the instantaneous pressure field computed at time $t=50$.

The analytical solution [62] is used to compute the convergence rate of the third-order FV-MK method reported on Table 5. Similar to previous observations on inviscid flows (see Section 4.1), present results are not very sensitive to the correlation parameter $\theta_{g}$. This is illustrated by the fact that all computations give comparable level of accuracy in the error of the pressure field. Moreover, the formal third-order accuracy of the method is recovered as shown in Table 5 and in Fig. 17. 

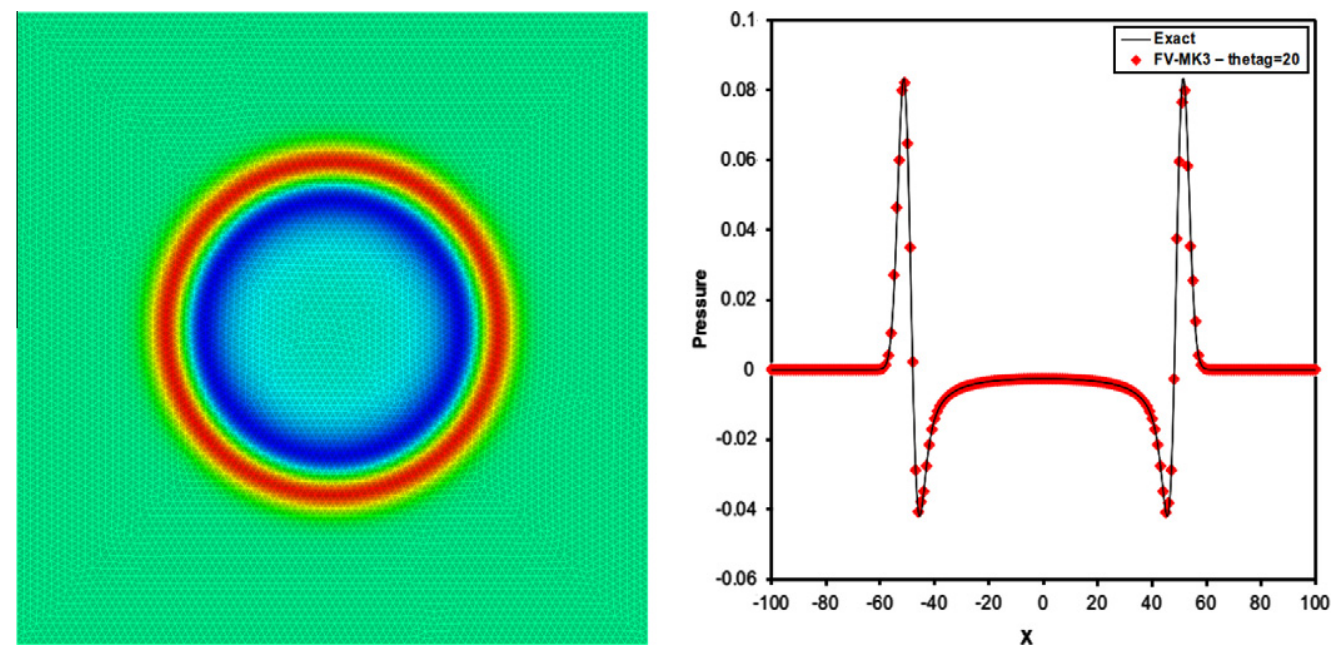

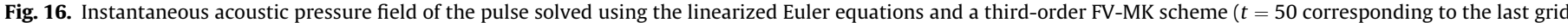
of Table (5) below).

Table 5

$L_{2}$-norms of error in pressure and corresponding convergence rates obtained for different values of the correlation parameter.

\begin{tabular}{|c|c|c|c|c|c|c|}
\hline \multirow[t]{2}{*}{ Cells } & \multicolumn{2}{|l|}{$\theta_{g}=10$} & \multicolumn{2}{|l|}{$\theta_{g}=15$} & \multicolumn{2}{|l|}{$\theta_{g}=20$} \\
\hline & $L_{2}$-error & Order & $L_{2}$-error & Order & $L_{2}$-error & Order \\
\hline 2020 & $8.213 \mathrm{E}-03$ & - & $8.803 \mathrm{E}-03$ & - & $6.101 \mathrm{E}-03$ & - \\
\hline 4548 & $2.171 \mathrm{E}-03$ & 3.28 & $2.361 \mathrm{E}-03$ & 3.24 & $1.757 \mathrm{E}-03$ & 3.07 \\
\hline 5608 & $1.574 \mathrm{E}-03$ & 3.07 & $1.720 \mathrm{E}-03$ & 3.02 & $1.237 \mathrm{E}-03$ & 3.35 \\
\hline 8102 & $7.771 \mathrm{E}-04$ & 3.84 & $8.964 \mathrm{E}-04$ & 3.54 & $7.254 \mathrm{E}-04$ & 2.90 \\
\hline 15470 & $2.913 \mathrm{E}-04$ & 3.03 & $3.346 \mathrm{E}-04$ & 3.05 & $2.664 \mathrm{E}-04$ & 3.10 \\
\hline
\end{tabular}

\subsection{Flow past a circular cylinder at $\operatorname{Re}_{D}=3900$}

In this example we show the application of the proposed scheme to a viscous unsteady flow problem illustrated by the unsteady 2D Navier-Stokes equations. The considered case concerns a detached flow around a cylinder at Mach 0.2 with a Reynolds number of 3900. The present computational results are compared to other numerical studies [63-65] and also to experiments $[66,67]$ in order to assess the accuracy of our approach. To solve this case, we employ the third-order order FV-MK scheme based on a Gaussian correlation function with $\theta_{g}=10$. This level of spatial accuracy was selected according to the results of $[68,69]$.

Fig. 18 depicts the unstructured triangular grid which has 150 elements on the surface around the cylinder and 200 points on the outer circular boundary. The corresponding total number of control volume is 35,000 . The far-field boundaries are $25 \mathrm{D}$ far from the cylinder ( $D$ being the diameter of the cylinder). The aerodynamic coefficients are computed using 30 vortex shedding cycles and the size of the non-dimensional time-step is $10^{-4}$.

Table 6 shows that the aerodynamic coefficients (namely the drag coefficient $\left(C_{d}\right)$, the back pressure coefficient $\left(C_{p b}\right)$ the separation angle $\theta$ and the Strouhal (St) number) present a global agreement with previous computations as far as we compare with 2D or poorly resolved 3D numerical simulations. Obviously, the values of $C_{d}$ and $C_{p b}$ are much too high compared with the experimental measurement since the three-dimensional effects, which strongly influence the near-wake, are not captured. On the contrary, the computed value of $S t$ seems to be less sensitive as already observed in [65].

The sequence of instantaneous vorticity fields presented in Fig. 19 exhibits the typical flow structure obtained on previous 2D numerical simulations [63].

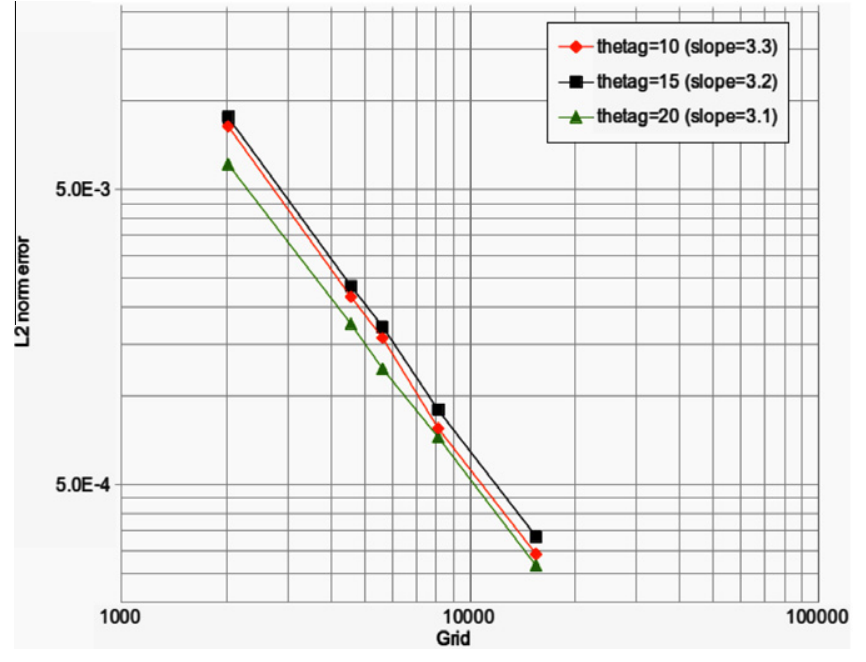

Fig. 17. $L_{2}$-norm of pressure error as a function of the grid size for the acoustic pulse problem computed with the third-order FV-MK scheme.

\subsection{Decay of compressible isotropic turbulence}

In this section we investigate the ability of the finite volume method based on moving Kriging interpolations to solve turbulent flows. To this end, we address the problem of compressible isotropic turbulence decay. This is a standard test case for numerical methods and turbulence models. In this example a third-order FV-MK method with exponential correlation is used with a complete cubic polynomial basis. Note that for this 3D case, we use 34 interpolation points to compute the derivatives at each centroid, and 42 interpolation points for the computation of viscous fluxes. The computational domain is the cube $[0,2 \pi]^{3}$, and periodic boundary conditions are used in all the directions. No explicit turbulence model is used in this example. We follow the idea presented in [70], where the dissipation introduced by the numerical scheme plays the role of the subgrid model. We follow the setup of one of the cases presented in [71]. The turbulence length scale is defined by selecting the initial three-dimensional energy spectrum as

$E_{3 D} \propto k^{4} \exp \left[-2\left(\frac{k}{k_{p}}\right)^{2}\right]$, 


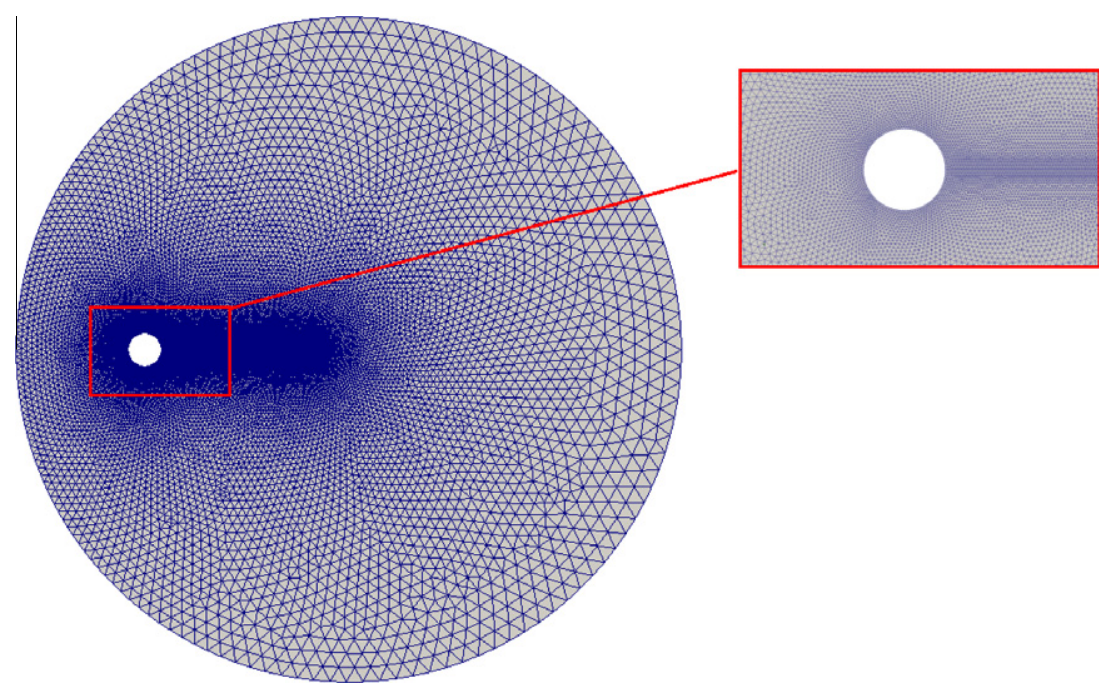

Fig. 18. Unstructured grid used for the unsteady computation past a circular cylinder at $R e=3900$.

Table 6

Third-order FV-MK results for the simulation of an unsteady flow past a circular cylinder at $R e=3900$.

\begin{tabular}{|c|c|c|c|c|c|c|}
\hline Ref. & Method & Dimension & $C_{d}$ & $-C_{p b}$ & $\theta$ & St \\
\hline Present & FV-MK3-G & $2 \mathrm{D}$ & 1.33 & 1.21 & 84.2 & 0.239 \\
\hline Young and Ooi [63] & URANS & $2 \mathrm{D}$ & 1.59 & 1.96 & - & 0.24 \\
\hline Lynch and Smith [64] & LES-RANS & $2 \mathrm{D}$ & 1.5 & - & 86.5 & 0.25 \\
\hline Young and Ooi [63] & LES & $3 \mathrm{D}\left(n_{z}=4\right)$ & 1.55 & 1.86 & - & 0.22 \\
\hline Breuer [65] & LES & $3 \mathrm{D}\left(n_{z}=64\right)$ & 1.016 & 0.94 & 87.4 & - \\
\hline Kravchenko and Moin [67] & Experiments & - & $0.99 \pm 0.05$ & $0.88 \pm 0.05$ & $86 \pm 2$ & $0.215 \pm 0.005$ \\
\hline
\end{tabular}
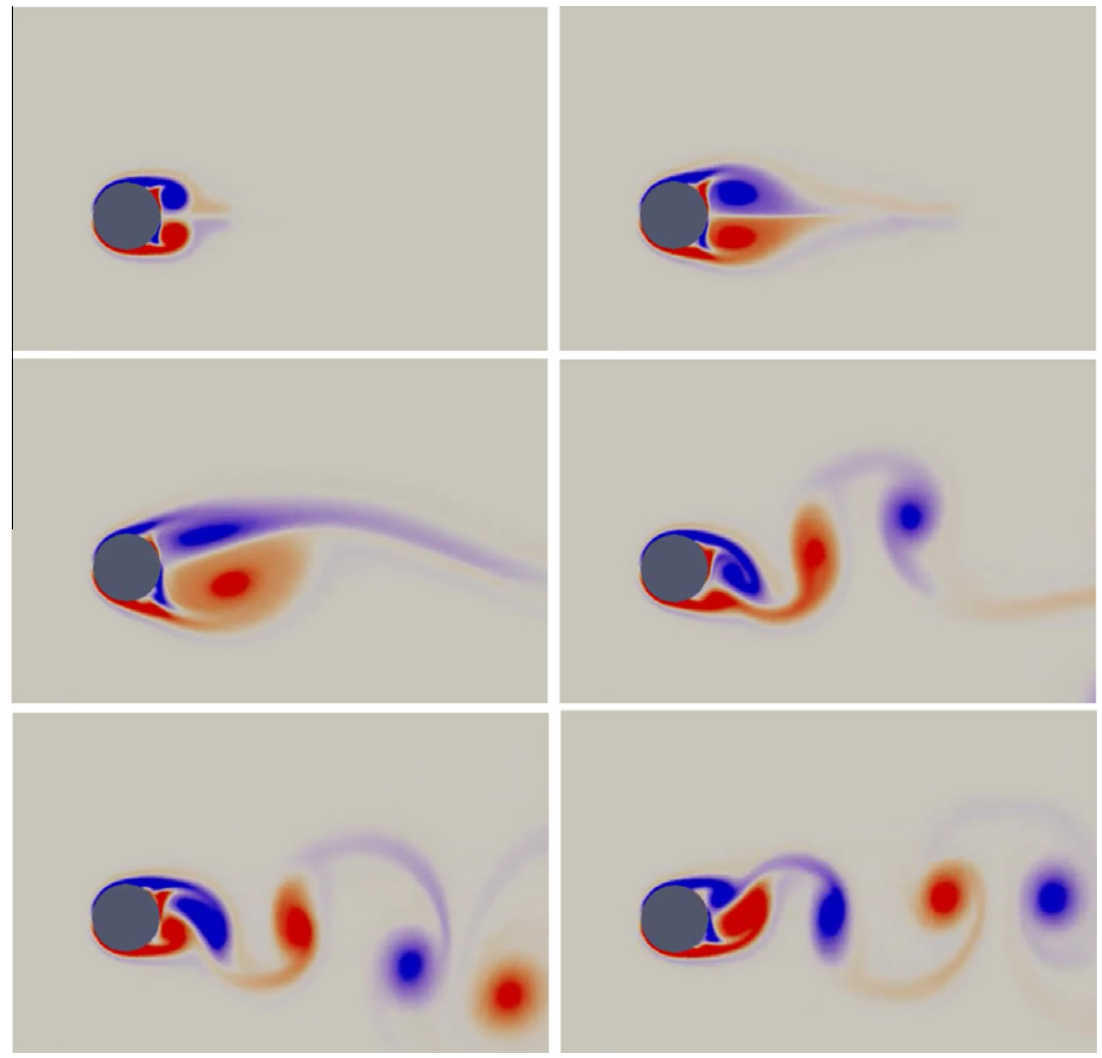

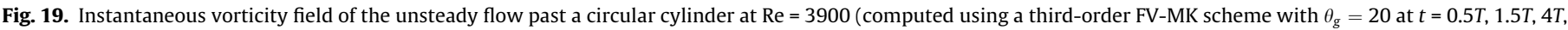
$4.75 T, 5.75 T$ and $10 T, T$ being the period of the vortex shedding). 

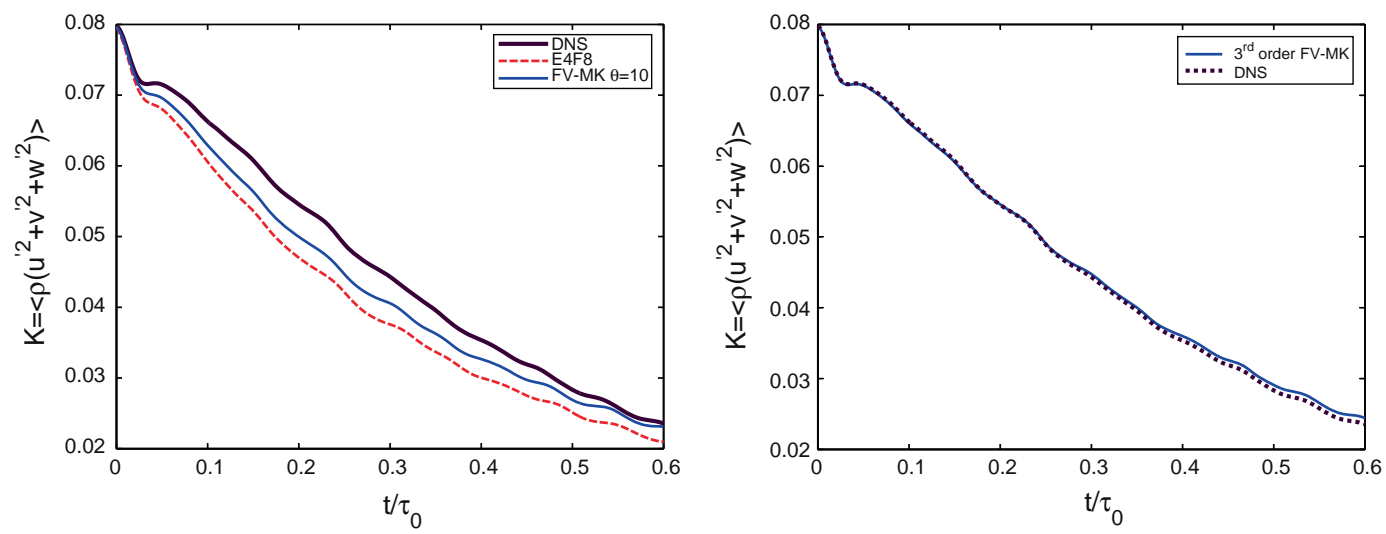

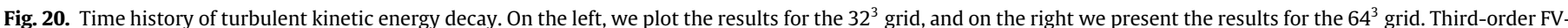
MK scheme with exponential correlation and $\theta_{g}=10$.
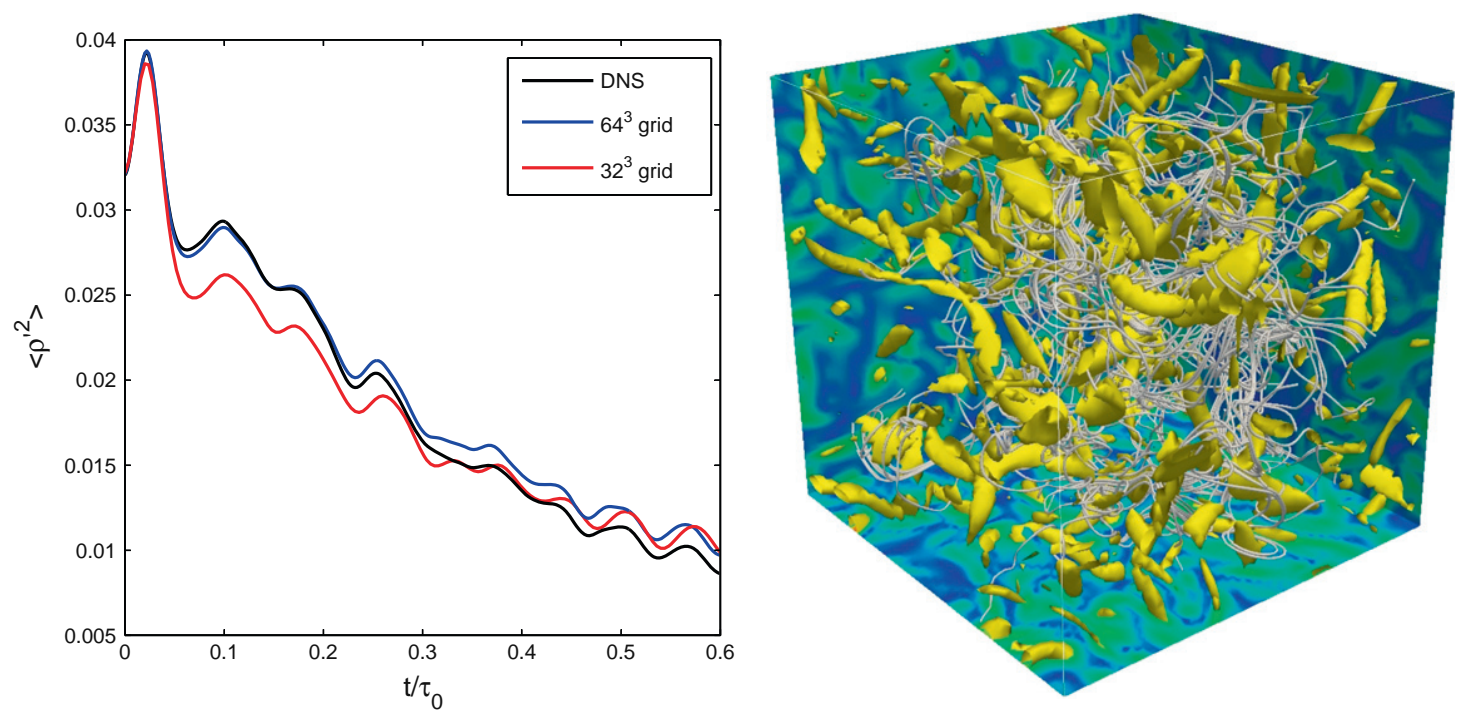

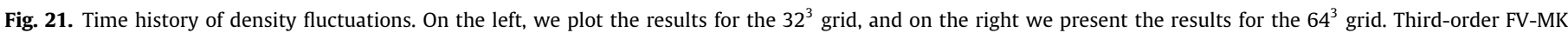
scheme with exponential correlation and $\theta_{g}=10$. The isovorticity surfaces and streamlines are shown in the right for the $64^{3}$ grid.
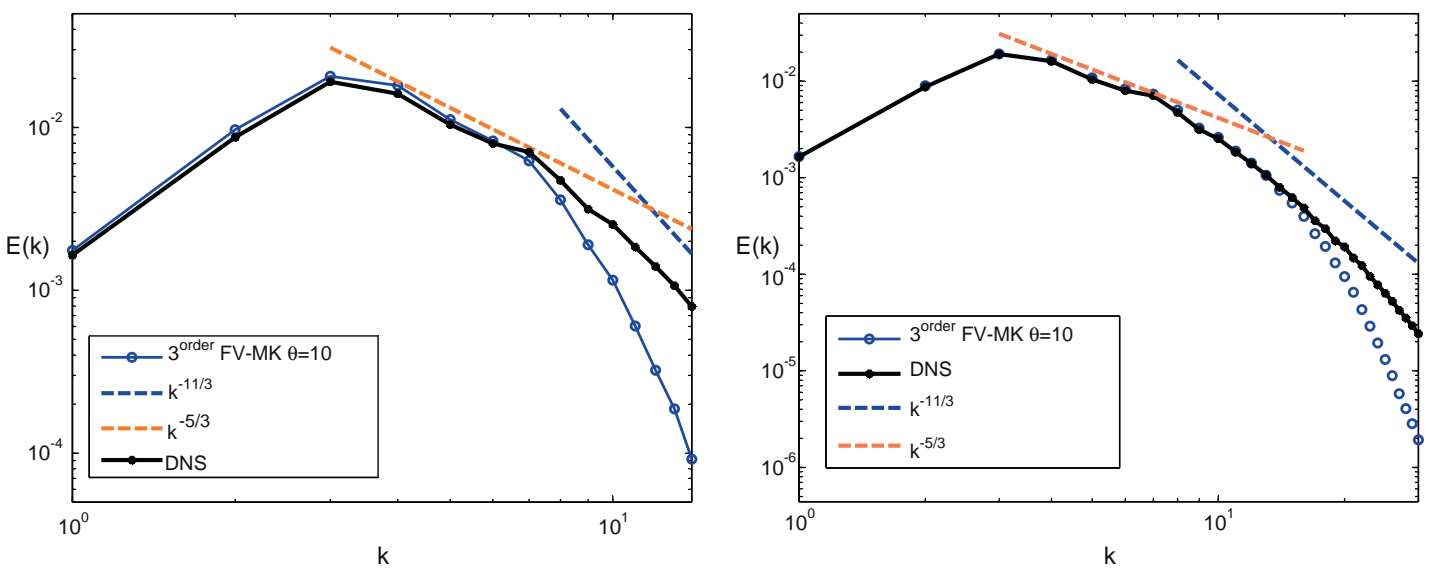

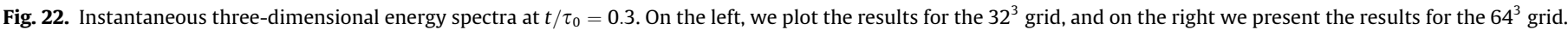
Third-order FV-MK scheme with exponential correlation and $\theta_{g}=10$. 
where $k_{p}=4$ is the wavenumber corresponding to the peak of the spectrum and $k$ is the wavenumber.

The parameter $\chi$ is defined as the ratio of compressible kinetic energy to the total turbulent kinetic energy [72]. It can be seen as an indicator of the level of compressibility of the flow, where $\chi=0$ corresponds to an incompressible flow. In the present case we choose $\chi=\left(q^{d} / q\right)^{2}=0.2$, where $q$ is the root mean square magnitude of the fluctuation velocity, and $q^{d}$ is the root mean square magnitude of the dilatational fluctuation velocity.

We have tested the method in two grids. One with $32^{3}$ elements and the other with $64^{3}$ elements. An explicit fourth-order RungeKutta scheme is used for time integration, with a time step of $\Delta t=0.05$. This value corresponds approximately to 250 time-steps per eddy turnover time $\left(\tau_{0}\right)$. The eddy turnover time is defined as the ratio of the turbulent kinetic energy to the dissipation rate based on the initial field. As a reference solution we use a computation on a $128^{3}$ grid, computed with sixth-order compact finite differences and an explicit Padé filter with parameter $\alpha=0.49$ [70].

Fig. 20 shows the decay of the turbulent kinetic energy in the two grids. We observe that the solution converges to the DNS as the grid is refined. For comparison purposes, we also show the results for a fourth-order centered finite difference method. Note that the results obtained for the third-order FV-MK method are also more accurate than the results obtained with an standard MUSCL third-order finite volume scheme [70]. Density fluctuations are plotted in Fig. 21. The results also converge to the DNS as the grid is refined. In this figure we also show the isovorticity surfaces and streamlines on the $64^{3}$ grid. Vortical structures such as worms or sheets are clearly identified.

The instantaneous three-dimensional energy spectra at $t / \tau_{0}=0.3$ is plotted in Fig. 22. The method is able to capture the two different slopes appearing of the three-dimensional energy spectrum. This result is predicted by the Eddy-Damped QuasiNormal Markovian Theory (EDQNM) [73].

\section{Conclusion}

A novel combination between a moving Kriging kernel reproducing approximation and a high-order finite volume method has been developed for the solution of viscous compressible flow on unstructured grids. A Taylor series expansion is considered to evaluate the value of the variables at the edges of the element. The numerical fluxes are computed at each quadrature points of the cell face. The successive derivatives of the continuous reconstruction field are expressed in terms of the derivatives of the moving Kriging shape functions evaluated at the cell centroids. In general, this step is cheaper than for other existing kernel reproducing approximations, since only the derivatives of the polynomial basis and of the correlation functions are involved in the reconstruction.

The moving Kriging finite volume method was first applied to the Ringleb flow problem. Expected theoretical orders of accuracy were recovered up to quadratic reconstructions. In particular, we have observed that the accuracy of the method is not excessively sensitive to the choice of the correlation parameter. Moreover, dramatic reduction in the production of spurious entropy were observed for the case of a subsonic flow past a NACA 0012 airfoil, in contrast with second-order least squares reconstructions. The results obtained for relatively large moving Kriging stencils, which are used in practice to enforce well-conditioning of the correlation matrix, show that robustness enhancement can be achieved without deteriorating the accuracy of the numerical scheme. Finally, the FV-MK approach was successfully deployed in the context of unsteady flows with applications to both wave propagation and viscous flow problems. Due to attractive features, such as robustness, flexibility, accuracy and easy implementation, we believe that the use of moving Kriging shape functions represents an interesting alternative to existing reproducing kernel approximations for the development of efficient high-order finite volume methods.

\section{Acknowledgements}

$\mathrm{X}$. Nogueira gratefully acknowledges the financial support from the Ministerio de Ciencia e Innovación (Grants \#DPI2009-14546C02-01 and \#DPI2010-16496), the R\&D projects of the Xunta de Galicia (Grants \#CN2011/002, \#PGDIT 09MDS00718PR and \#PGDIT09REM005118PR, co-financed with FEDER funds), and from the Universidade da Coruna.

\section{References}

[1] Z. Wang, High-order methods for the Euler and Navier-Stokes equations on unstructured grids, Prog. Aerosp. Sci. 43 (2007) 1-4.

[2] H. Luo, J. Baum, R. Lohner, On the computation of steady-state compressible flows using a discontinuous Galerkin method, Int. J. Numer. Methods Engrg. 73 (2008) 597-623.

[3] L. Krivodonova, M. Berger, High-order accurate implementation of solid wall boundary conditions in curved geometries, J. Comput. Phys. 211 (2006) 492512.

[4] T. Barth, P. Frederickson, Higher-order Solution of the Euler Equations on Unstructured Grids Using Quadratic Reconstruction, AIAA Paper 90-0013, 1990.

[5] A. Godfrey, C. Mitchell, R. Walters, Practical aspects of spatially high-order accurate methods, AIAA J. 31 (1993) 1634-1642.

[6] T. Barth, Recent Developments in High Order $k$-exact Reconstruction on Unstructured Meshes, AIAA Paper 93-0668, 1990.

[7] M. Delanaye, P. Geuzaine, J. Essers, The quadratic reconstruction finite volume scheme: an attractive sequel to linear reconstruction, used on unstructured adaptive meshes, Lect. Notes Phys. 490 (1997) 617-622.

[8] C. Ollivier-Gooch, C.V. Altena, A high-order accurate unstructured mesh finitevolume scheme for the advection-diffusion equation, J. Comput. Phys. 181 (2002) 729-752.

[9] D. Vigneron, J.-M. Vaassen, J.-A. Essers, An implicit high order cell-centered finite volume scheme for the solution of three-dimensional Navier-Stokes equations on unstructured grids, in: Third MIT Conference on Computational Fluid and Solid Mechanics, June 23-27, MA, USA, 2003.

[10] A. Nejat, C. Ollivier-Gooch, A high-order accurate unstructured finite volume Newton-Krylov algorithm for inviscid compressible flows, J. Comput. Phys. 227 (2008) 2582-2609.

[11] C. Ollivier-Gooch, A. Nejat, K. Michalak, Obtaining and verifying high-order unstructured finite volume solution to the Euler equations, AIAA J. 47 (2009) 2105-2120.

[12] R. Abgrall, On essentially non-oscillatory schemes on unstructured meshes: analysis and implementation, J. Comput. Phys. 114 (1994) 45-58.

[13] C. Ollivier-Gooch, Quasi-ENO schemes for unstructured meshes based on unlimited data-dependent least-squares reconstruction, J. Comput. Phys. 133 (1997) 6-17.

[14] O. Friedrich, Weighted essentially non-oscillatory schemes for the interpolation of mean values on unstructured grids, J. Comput. Phys. 144 (1998) 194-212.

[15] C. Hu, C. Shu, Weighted essentially non-oscillatory schemes on triangular meshes, J. Comput. Phys. 150 (1999) 97-127.

[16] C.-W. Shu, High-order finite difference and finite volume WENO schemes and discontinuous Galerkin methods for CFD, Int. J. Comput. Fluid Dynam. 17 (2003) 107-118.

[17] M. Dumbser, M. Kaser, V. Titarev, E. Toro, Quadrature-free non-oscillatory finite volume schemes on unstructured meshes for nonlinear hyperbolic systems, J. Comput. Phys. 226 (2007) 204-243.

[18] P. Lancaster, K. Salkauskas, Surfaces generated by moving least squares methods, Math. Comput. 87 (1981) 141-158.

[19] W. Liu, S. Li, T. Belytschko, Moving least-squares reproducing kernel methods. I. Methodology and convergence, Comput. Methods Appl. Mech. Engrg. 143 (1997) 113-154

[20] L. Cueto-Felgueroso, I. Colominas, J. Fe, F. Navarrina, M. Casteleiro, High-order finite volume schemes on unstructured grids using moving least-squares reconstruction. application to shallow water dynamics, Int. J. Numer. Methods Engrg. 65 (2006) 295-331.

[21] X. Nogueira, I. Colominas, L. Cueto-Felgueroso, S. Khelladi, On the simulation of wave propagation with a higher-order finite volume scheme based on reproducing kernel methods, Comput. Methods Appl. Mech. Engrg. 199 (2010) 1471-1490.

[22] X. Nogueira, I. Colominas, L. Cueto-Felgueroso, S. Khelladi, F. Navarrina, M. Casteleiro, Resolution of computational aeroacoustics problems on unstructured grids with a higher-order finite volume scheme, J. Comput. Appl. Math. 234 (2010) 2089-2097. 
[23] L. Cueto-Felgueroso, I. Colominas, X. Nogueira, F. Navarrina, M. Casteleiro, Finite-volume solvers and moving least-squares approximations for the compressible Navier-Stokes equations on unstructured grids, Comput. Methods Appl. Mech. Engrg. 196 (2007) 4712-4736.

[24] X. Nogueira, L. Cueto-Felgueroso, I. Colominas, H. Gomez, F. Navarrina, M. Casteleiro, On the accuracy of finite volume and discontinuous Galerkin discretizations for compressible flow on unstructured grids, Int. J. Numer. Methods Engrg. 78 (2009) 1553-1584.

[25] T. Most, C. Bucher, A moving least squares weighting function for the elementfree Galerkin method which almost fulfills essential boundary conditions, Struct. Engrg. Mech. 21 (2005) 315-332.

[26] T. Most, C. Bucher, New concepts for moving least squares: an interpolating non-singular weighting function and weighted nodal least squares, Engrg. Anal. Bound. Elem. 32 (2008) 461-470.

[27] S. Lophaven, H. Nielsen, J. Sndergaard, Dace: A Matlab Kriging Toolbox Technical Report IMM-TR-2002-12, Informatics and Mathematical Modelling, Technical University of Denmark, DK-2800 Kgs. Lyngby, Denmark, 2002.

[28] N. Sukumar, R. Wright, Overview and construction of meshfree basis functions: from moving least squares to entropy approximants, Int. J. Numer. Methods Engrg. 70 (2007) 181-205.

[29] K. Dai, G. Liu, K. Lim, Y. Gu, Comparison between the radial point interpolation and the Kriging interpolation used in meshfree methods, Comput. Mech. 32 (2003) 60-70.

[30] R. Hardy, Theory and applications of the multiquadrics-biharmonic method (20 years of discovery 1968-1988), Comput. Math. Appl. 19 (1990) 163-208.

[31] J. Wang, G. Liu, A point interpolation meshless method based on radial basis functions, Int. J. Numer. Methods Fluids 54 (2002) 1623-1648.

[32] M. Arroyo, M. Ortiz, Local maximum entropy approximation schemes: a seamless bridge between finite elements and meshfree methods, Int. J. Numer. Methods Engrg. 65 (2006) 2167-2202.

[33] L. Stein, Interpolation of Spatial Data: Some Theory for Kriging, first ed., Springer, 1999.

[34] T. Simpson, T. Mauery, J. Korte, F. Mistree, Kriging models for global approximation in simulation-based multidisciplinary design optimization, AIAA J. 39 (2001) 2233-2241.

[35] S. Sakataa, F. Ashidaa, M. Zakob, Structural optimization using Kriging approximation, Comput. Methods Appl. Mech. Engrg. 192 (2003) 923-939.

[36] L. Gu, Moving Kriging interpolation and element-free Galerkin method, Int. J. Numer. Methods Engrg. 56 (2003) 1-11.

[37] H. Li, Q. Wang, K. Lam, Computational mechanics, Comput. Methods Appl. Mech. Engrg. 193 (2004) 2599-2619.

[38] P. Tongsuk, W. Kanok-Nukulchai, Further investigation of elementfree Galerkin method using moving Kriging interpolation, Int. J. Comput. Methods 1 (2004) 1-21.

[39] F. Wong, W. Kanok-Nukulchai, A Kriging-based finite element method for analyses of shell structures, in: WCCM8, ECCOMAS 2008, June 30-July 5, Venice, Italy, 2008.

[40] T. Bui, T. Nguyen, H. Nguyen-Dang, A moving Kriging interpolation-based meshless method for numerical simulation of Kirchhoff plate problems, Int. J. Numer. Methods Engrg. 77 (2009) 1371-1395.

[41] F. Wong, W. Kanok-Nukulchai, Kriging-based finite element method: elementby-element Kriging interpolation, Civil Engrg. Dimension 11 (1) (2009) 15-22.

[42] F. Wong, H. Syamsoeyadi, Kriging-based timoshenko beam element for static and free vibration analyses, Civil Engrg. Dimension 13 (2011) 42-49.

[43] T. Bui, M. Nguyen, Eigenvalue analysis of thin plate with complicated shapes by a novel mesh-free method, Int. J. Appl. Mech. 3 (2011) 21-46.

[44] A. Shaw, S. Bendapudi, D. Roy, A Kriging-based error-reproducing and interpolating kernel method for improved mesh-free approximations, Int. J. Numer. Methods Engrg. 73 (2008) 1434-1467.

[45] P. Batten, N. Clarke, C. Lambert, D. Causon, On the choice of wavespeeds for the HLLC Riemann solver, SIAM J. Sci. Comput. 18 (1997) 1553-1570.

[46] P. Batten, M. Leschziner, U. Goldberg, Average-state jacobians and implicit methods for compressible viscous and turbulent flows, J. Comput. Phys. 137 (1997) 38-78.

[47] C.-W. Shu, S. Osher, Efficient implementation of essentially non-oscillatory shock-capturing schemes, J. Comput. Phys. 77 (1988) 439.

[48] P. Jawahar, H. Kamath, A high-resolution procedure for Euler and NavierStokes computations on unstructured grids, J. Comput. Phys. 164 (2000) 165203.
[49] S. Khelladi, X. Nogueira, F. Bakir, I. Colominas, Toward a higher-order unsteady finite volume solver based on reproducing kernel particle method, Comput Methods Appl. Mech. Engrg. 200 (2011) 2348-2362.

[50] A. Huerta, Y. Vidal, P. Villon, Pseudo-divergence-free element free galerkin method for incompressible fluid flow, computer methods in applied mechanics and engineering, Comput. Methods Appl. Mech. Engrg. 2004 (2004) 1119-1136.

[51] S. Chenoweth, J. Soria, A. Ooi, A singularity-avoiding moving-least-squares scheme for two-dimensional unstructured meshes, J. Comput. Phys. 228 (2009) 5592-5619.

[52] X. Nogueira, L. Cueto-Felgueroso, I. Colominas, F. Navarrina, M. Casteleiro, A new shock-capturing technique based on moving least squares for higherorder numerical schemes on unstructured grids, Comput. Methods Appl. Mech. Engrg. 199 (2010) 2544-2548.

[53] F. Bassi, S. Rebay, High-order accurate discontinuous finite element solution of the 2d Euler equations, J. Comput. Phys. 138 (1997) 251-285.

[54] K. Fidkowski, D. Darmofal, Development of a Higher-order Solver for Aerodynamic Applications, AIAA Paper 2004-0436, 2004.

[55] V. Dolejsi, M. Feistauer, A semi-implicit discontinuous Galerkin finite element method for the numerical solution of inviscid compressible flow, J. Comput. Phys. 198 (2004) 727-746.

[56] L. Ivan, C. Groth, High-order Central ENO Finite-volume Scheme with Adaptive Mesh Refinement, AIAA Paper 2007-4323, 2007.

[57] G. Chiocchia, Exact Solutions to Transonic and Supersonic Flows, AGARD-AR211, Advisory Report, 1985.

[58] H. Viviand, Numerical Solutions of Two-dimensional Reference Test Cases, AGARD-AR-211, Advisory Report, 1985.

[59] M. Delanaye, J. Essers, Polynomial reconstruction finite volume scheme for compressible flows on unstructured adaptive grids, AIAA J. 35 (1997) 631-639.

[60] V. Venkatakrishnan, D. Mavriplis, Implicit method for the computation of unsteady flows on unstructured grids, J. Comput. Phys. 127 (1996) 380-397.

[61] J. Berland, C. Bogey, C. Bailly, Low-dissipation and low-dispersion fourth-order Runge-Kutta algorithm, Comput. Fluids 35 (10) (2006) 1459-1463.

[62] C. Tam, J. Webb, Dispersion-relation-preserving finite difference schemes for computational aeroacoustics, J. Comput. Phys. 107 (1993) 262-281.

63] M. Young, A. Ooi, Comparative assessment of LES and URANS for flow over a cylinder at a Reynolds number of 3900, in: 16th Australian Fluid Mechanics Conference, Gold Coast, Australia, 2-7 December 2007.

[64] C.E. Lynch, M.J. Smith, Hybrid RANS-LES turbulence models on unstructured grids, in: AIAA Paper 2008-3854, 38th Fluid Dynamics Conference and Exhibit, Seattle, Washington, 23-26 June 2008.

[65] M. Breuer, Large eddy simulation of the subcritical flow past a circular cylinder: numerical and modeling aspects, Int. J. Numer. Methods Fluids 28 (1998) 1281-1302.

[66] J. Son, T. Hanratty, Velocity gradients at the wall for flow around a cylinder at Reynolds numbers from $5 \times 10^{3}$ to $10^{5}$, J. Fluid Mech. 35 (1969) 353-368.

[67] A.G. Kravchenko, P. Moin, Numerical studies of flow over a circular cylinder at $R e_{d}=3900$, Phys. Fluids 12 (2) (2000) 403-417.

[68] X. Nogueira, L. Cueto-Felgueroso, I. Colominas, H. Gómez, Implicit large eddy simulation of non-wall-bounded turbulent flows based on the multiscale properties of a high-order finite volume method, Comput. Methods Appl. Mech. Engrg. 199 (9-12) (2010) 615-624.

[69] S. Khelladi, X. Nogueira, F. Bakir, I. Colominas, Toward a higher order unsteady finite volume solver based on reproducing kernel methods, Comput. Methods Appl. Mech. Engrg. 200 (29-32) (2011) 2348-2362.

[70] M.R. Visbal, D.P. Rizzeta, Large-eddy simulation on curvilinear grids using compact differencing and filtering schemes, J. Fluids Engrg. 124 (2002) 836847.

[71] E.T. Spyropoulos, G.A. Blaisdell, Evaluation of the dynamic model for simulations of compressible decaying isotropic turbulence, AIAA J. 34 (1996) 990-998.

[72] S. Sarkar, G. Erlebacher, M.Y. Hussaini, H.O. Kreiss, The analysis and modelling of dilatational terms in compressible turbulence, J. Fluid Mech. 227 (1991) 473-493.

[73] M.Y. Hussaini, On Large-Eddy Simulation of Compressible Flows, AIAA Paper 1998-2802, 1998. 